\title{
Unified treatment of nonlinear optical force in laser trapping of dielectric particles of varying sizes
}

\author{
Anita Devi ${ }^{1}$ and Arijit K. De $\odot^{2, *}$ \\ ${ }^{1}$ Department of Physical Sciences, Indian Institute of Science Education and Research (IISER) Mohali, \\ Knowledge City, Sector 81, SAS Nagar, Punjab 140306, India \\ ${ }^{2}$ Department of Chemical Sciences, Indian Institute of Science Education and Research (IISER) Mohali, \\ Knowledge City, Sector 81, SAS Nagar, Punjab 140306, India
}

(Received 3 November 2020; accepted 26 January 2021; published 20 July 2021)

\begin{abstract}
Optical trapping using laser tweezer has revolutionized the field of force spectroscopy having enormous applications in biological manipulation. While a number of theories were developed for particles of different sizes to estimate trapping force under continuous-wave excitation, they were not under short pulsed excitation which leads to nonlinear optical force. Here, we present a comparative study of various theories and provide a unified description for laser trapping under femtosecond pulsed excitation. Numerical results show that exact Mie theory (EMT) can provide a precise qualitative and quantitative prediction of trapping force when optical Kerr effect is included. Moreover, we also show how Mie interference phenomena, leading to observation of Fano resonance, are naturally captured within EMT. Thus, our findings pave the way for potential far-reaching applications in the accurate numerical estimation of nonlinear optical force on arbitrary-sized spherical dielectric particles.
\end{abstract}

DOI: 10.1103/PhysRevResearch.3.033074

An optical tweezer (OT) is a powerful tool to manipulate micron- to nanometer-sized objects. Ashkin discovered OT in 1970, contemplating the concept of radiation pressure [1]. OT has been extensively utilized for biological manipulations over decades. Both theoretical and experimental approaches have been studied thoroughly, which continues to expand even today [2-7]. For the past few decades, tremendous work has been done for OT using continuous-wave (cw) excitation [5-7]. In contrast to this, very few experimental and theoretical studies have been performed with femtosecond pulses [8-20]. It has been demonstrated that the nanoparticles could experience $100 \mathrm{pN}$ force at lower average power [15-18,21,22] because of the higher peak intensity of pulse excitation than $\mathrm{cw}$ excitation. It is quite remarkable to notice that under pulsed excitation, no considerable change is observed in trapping efficiency for a micron-sized silica particle [9]. However, for micron-sized polystyrene particles, a substantial change in trapping efficiency is theoretically estimated due to the significant contribution of nonlinear optical effects $[23,24]$. For nanoparticles, trapping is quite strenuous under $\mathrm{cw}$ excitations because with the decrease in particle size, Brownian motion increases drastically, and very high average power will be required to create a deeper potential well. Nevertheless, high average power comes with other repercussions. Therefore, it is argued that pulsed excitation might yield a much better trapping efficiency due to instantaneous repetitive momentum transfer [25]. However, there

\footnotetext{
*akde@iisermohali.ac.in

Published by the American Physical Society under the terms of the Creative Commons Attribution 4.0 International license. Further distribution of this work must maintain attribution to the author(s) and the published article's title, journal citation, and DOI.
}

is no concrete experimental demonstration to date. Theoretically, a substantial change in trapping efficiency has been shown for dielectric [26,27] and metallic [28-30] nanoparticles. Subsequent studies showed that using pulsed excitation, hollow-core nanoparticles can be trapped which are challenging to be trapped under $\mathrm{cw}$ excitation [31,32]. All the previous studies were performed using dipole [26], geometric optics (GO) [23,24], and generalized Lorenz Mie theory (GLMT) [27] approximations. These theories have their limitations in accurately calculating the total force for arbitrary size particles. It was later predicted that exact Mie theory (EMT) might be one of the most optimal methods applicable for all size limits of particles under cw excitation [5,33]. However, no general theory has been predicted till now for pulsed excitation. In this paper, we present an accurate method of calculating force acting on the arbitrary size particle by comparing EMT results with dipole, GLMT, and GO approximations under pulsed excitations taking the Kerr effect into account.

In the simulation, it is assumed that the particle is spherical, isotropic, homogeenous, and nonmagnetic. Here, all the force simulations are presented along the axial direction $(z)$ because this is the most crucial direction for optical trapping because both scattering and gradient forces are experienced by the particle, whereas along the radial direction, only gradient force is present which results in stabilizing the trap. Depending upon the particle radius $(a)$ and wavelength of the trapping beam $(\lambda)$, there are three different regimes: the Rayleigh regime (where $a \ll \lambda$ ), the geometric optics (GO) regime (where $a \gg \lambda$ ), and the Mie regime (where $a \approx \lambda$ ) [5]. A detailed discussion on calculating the force using EMT is provided below, whereas dipole, GLMT, and GO approximations are discussed in Sec. 1 of the Appendix. Please note that we use paraxial approximation and localized approximation for dipole theory and GLMT, respectively. 
EMT is derived from the Maxwell stress tensor theory in terms of trapping efficiency by considering the spherical surface at infinity [33]. In simplified form, force expression along the axial direction can be written as [34]

$$
F^{\mathrm{EMT}}=\frac{n^{w}}{c} P_{\text {peak } / \mathrm{avg}} Q .
$$

Here, $n^{w}$ is the refractive index (RI) of the medium (water), $c$ is the speed of light, $P_{\text {peak/avg }}$ is the peak power for pulsed and average power for $\mathrm{cw}$ excitations, and $Q$ is the trapping efficiency which can be expressed as [33] $Q=Q_{e}+Q_{s}$. $Q_{e}$ is contributed by $|E|^{2}$ and $|B|^{2}$, where $E=E_{0}+E_{s}$ is total electric field and $B=B_{0}+B_{s}$ is total magnetic field [33], whereas, $Q_{s}$ is contributed by the remaining terms (such as $E_{0} B_{s}, E_{s} B_{0}$, etc.). In trapping efficiency, the contribution of $E$ and $B$ are not quite evident, as they inherently contribute through Mie scattering coefficients (MSCs) and beam shaping coefficients (BSCs). The trapping efficiency can be written as a summation of $Q_{e}$ and $Q_{s}$, which can be expressed as [33]

$$
\begin{aligned}
Q_{e}= & \frac{4 \gamma^{2}}{A} \operatorname{Re}\left[\sum_{n}(2 n+1) S_{n, \mathrm{EMT}}^{(1)} G_{n} G_{n}^{\prime *}\right] \\
= & \frac{4 \gamma^{2}}{A} \sum_{n}(2 n+1)\left(\operatorname{Re}\left[S_{n, \mathrm{EMT}}^{(1)}\right] \operatorname{Re}\left[G_{n} G_{n}^{\prime *}\right]-\operatorname{Im}\left[S_{n, \mathrm{EMT}}^{(1)}\right] \operatorname{Im}\left[G_{n} G_{n}^{\prime *}\right]\right), \\
Q_{s}= & -\frac{8 \gamma^{2}}{A} \operatorname{Re}\left[\sum_{n}\left(\frac{n(n+2)}{n+1} S_{n, \mathrm{EMT}}^{(2)} G_{n} G_{n+1}^{*}+\frac{2 n+1}{n(n+1)} S_{n, \mathrm{EMT}}^{(3)} G_{n} G_{n}^{*}\right)\right] \\
= & -\frac{8 \gamma^{2}}{A} \sum_{n}\left(\frac{n(n+2)}{n+1}\left(\operatorname{Re}\left[S_{n, \mathrm{EMT}}^{(2)}\right] \operatorname{Re}\left[G_{n} G_{n+1}^{*}\right]-\operatorname{Im}\left[S_{n, \mathrm{EMT}}^{(2)}\right] \operatorname{Im}\left[G_{n} G_{n+1}^{*}\right]\right)\right. \\
& \left.+\frac{2 n+1}{n(n+1)}\left(\operatorname{Re}\left[S_{n, \mathrm{EMT}}^{(3)}\right] \operatorname{Re}\left[G_{n} G_{n}^{*}\right]-\operatorname{Im}\left[S_{n, \mathrm{EMT}}^{(3)}\right] \operatorname{Im}\left[G_{n} G_{n}^{*}\right]\right)\right),
\end{aligned}
$$

where $A$ is the fraction of power passing through the objective, $\gamma$ is the ratio of objective focal length to the beam waist which can be calculated as $\gamma=\sqrt{-\frac{\ln (1-A)}{2 \sin ^{2}\left(\phi_{\max }\right)}}$, where $\phi_{\max }$ is the maximum focusing angle with the beam axis. $S_{n, \mathrm{EMT}}^{(1)}, S_{n, \mathrm{EMT}}^{(2)}$ and $S_{n, \text { EMT }}^{(3)}$ are scattering coefficients which can be expressed as

$$
\begin{aligned}
& S_{n, \mathrm{EMT}}^{(1)}=a_{n}+b_{n}, \\
& S_{n, \mathrm{EMT}}^{(2)}=a_{n} a_{n+1}^{*}+b_{n} b_{n+1}^{*}, \\
& S_{n, \mathrm{EMT}}^{(3)}=a_{n} b_{n}^{*},
\end{aligned}
$$

where, $a_{n}$ and $b_{n}$ are MSCs which are the functions of negative and positive half integer spherical Bessel and Ricatti-Bessel functions:

$$
\begin{gathered}
\psi_{n}(x)=\left(\frac{\pi x}{2}\right)^{1 / 2} J_{n+1 / 2}(x) \\
\xi_{n}(x)=\left(\frac{\pi x}{2}\right)^{1 / 2} J_{n+1 / 2}(x)+i(-1)^{n}\left(\frac{\pi x}{2}\right)^{1 / 2} J_{-n-1 / 2}(x),
\end{gathered}
$$

where the variable $x$ is either $\alpha=k \times a$ or $\beta=M \times \alpha$; here $M=\frac{n^{P}}{n^{w}}$ is the relative refractive index (RI) of the particle to the surrounding medium (here, $n^{p}$ is RI of the particle), and $k=\frac{2 \pi}{\lambda}$ is the propagating wave vector. The MSCs expression can be written as $[34,35]$

$$
\begin{aligned}
a_{n} & =\left(\frac{\psi_{n}(\alpha) \psi_{n}^{\prime}(\beta)-M \psi_{n}^{\prime}{ }_{n}(\alpha) \psi_{n}(\beta)}{\xi_{n}(\alpha) \psi_{n}^{\prime}(\beta)-M \xi_{n}^{\prime}(\alpha) \psi_{n}(\beta)}\right), \\
b_{n} & =\left(\frac{M \psi_{n}(\alpha) \psi_{n}^{\prime}(\beta)-\psi_{n}^{\prime}(\alpha) \psi_{n}(\beta)}{M \xi_{n}(\alpha) \psi^{\prime}{ }_{n}(\beta)-\xi_{n}^{\prime}(\alpha) \psi_{n}(\beta)}\right) .
\end{aligned}
$$

BSCs $\left(G_{n}\right)$ can be written as [33]

$$
\begin{gathered}
G_{n}=\int_{0}^{\phi_{\max }} d \phi \sin \phi \sqrt{\cos \phi} e^{-\gamma^{2} \sin ^{2} \phi+i \delta \cos \phi} d_{1,1}^{n}(\phi), \\
G_{n}^{\prime}=-i \frac{\partial G_{n}}{\partial \delta},
\end{gathered}
$$

where $\delta=k \times z$ ( $z$ corresponds to the axial position of the particle), and $d_{1,1}^{n}(\phi)$ is the matrix element of finite rotations, which can be written as [36]

$$
\begin{aligned}
d_{1,1}^{n}(\phi)= & \frac{1}{(2 n+1)}\left(\frac{P_{n-1}(\cos \phi)-P_{n+1}(\cos \phi)}{1-\cos ^{2} \phi}(1-\cos \phi)\right. \\
& \left.+(2 n+1) P_{n}(\cos \phi)\right),
\end{aligned}
$$

where $P_{j}(\cos \phi)$ are Legendre function of the first kind; $j$ is $n$, $n-1, n+1$ in the above equation.

All theoretical simulations are done with Mathematica (versions 10.0.0.0 and 11.0.0.0, Wolfram Research, Inc.) and MATLAB (R2017a, MathWorks, Inc.).

Nonlinearity (optical Kerr effect) is incorporated through the second-order nonlinear RI which can be expressed as $n^{w / p}=n_{0}^{w / p}+n_{2}^{w / p} \times I_{\text {peak/avg }}(z)$; here, $n_{0}^{w / p}$ and $n_{2}^{w / p}$ are the linear and nonlinear RI of the medium/particle, respectively. Water has a very small nonlinear RI so it can be neglected for both cw and pulsed excitation at low average power [26]. Therefore, we considered

$$
\begin{aligned}
n^{w / p} & \approx n_{0}^{w / p}(\text { under cw excitation }), \\
n^{w} & \approx n_{0}^{w}(\text { under pulsed excitation }), \\
n^{p} & =n_{0}^{p}+n_{2}^{p} \times I_{\text {avg } / \text { peak }}(z)(\text { under pulsed excitation }) .
\end{aligned}
$$



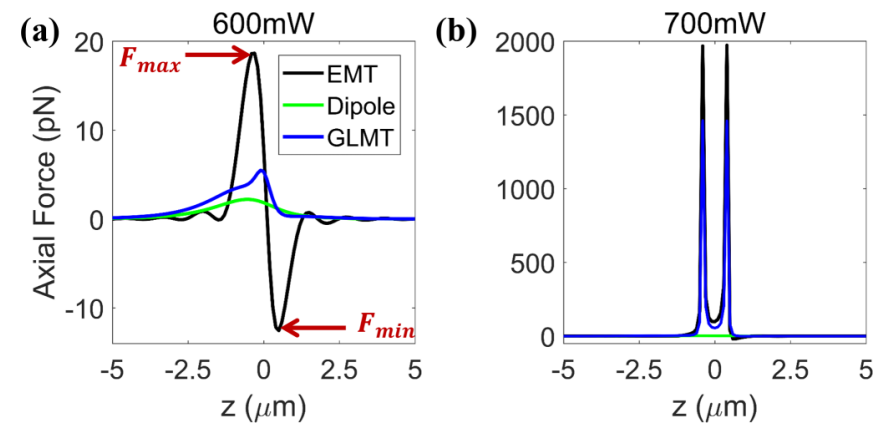

FIG. 1. Plots of trapping force against axial direction using dipole approximation, GLMT, and EMT for 40-nm particle size at (a) $600 \mathrm{~mW}$ and (b) $700 \mathrm{~mW}$ average power under pulsed excitation. Black, blue, green: EMT, GLMT, dipole approximation, respectively. Just for clear visualization, we multiplied the dipole and GLMT approximations force curves by a factor of 10 .

$I_{\text {peak/avg }}(z)$ is the peak or average intensity of focused Gaussian beam under pulsed or cw excitation, the mathematical form given in Sec. 1 a of the Appendix. All the parameters used in the simulation are given in Sec. 2 of the Appendix. Please note that by "dimension", we mean the radius of the particle.

The force expressions for EMT are strongly dependent upon BSCs [Eq. (7)] and MSCs [Eq. (6)]. MSCs depend upon $\alpha=k \times a$ and relative RI $(M)$ through Bessel and Hankel functions. However, in EMT, BSCs are strongly dependent upon $\phi_{\max }$ (discussed in Sec. 3 of the Appendix), $z$ (axial position), and summation over $n$ for Legendre and exponential functions. A small change in these parameters can lead to a pronounced change in the force acting on the particle. A detailed discussion on the contribution of MSCs and BSCs in the force calculations under both $\mathrm{cw}$ and pulsed excitation is given in Secs. 4 and 5 of the Appendix. For all particle size limits, under $\mathrm{cw}$ excitation, a change (increase or decrease) in average power leads to a change (increase or decrease) in the magnitude of force maxima $\left[F_{\max }\right.$; marked in Fig. 1(a)], force minima $\left[F_{\min }\right.$; marked in Fig. $\left.1(\mathrm{a})\right]$, and trap stiffness ( $k_{\text {stiffness }}$; discussed in Sec. 6 of the Appendix) in the same proportion, whereas no change in the nature of the force curve is observed because the analytical expression of force is directly proportional to the average power.

For nanoparticles, the magnitude of $F_{\max }, F_{\min }$, and $k_{\text {stiffness }}$ in EMT differ from those in other theories and increasing particle size increases the difference under both $\mathrm{cw}$ and pulsed excitations, as discussed in Sec. 7 of the Appendix. This might be due to the fact that EMT incorporates all the surface effects and calculations are done for tight focusing conditions that are missing in other theories. Overall, these theories agree qualitatively but disagree quantitatively. Quite interestingly, under pulsed excitation, at high average power, EMT reveals the occurrence of nonlinear phenomena which originate from MSCs [37], as shown in Fig. 1(b). This nonlinear phenomenon is also known as Fano resonance which appears in any medium due to the interference between transverse electric and magnetic fields [37,38]. Also, from the values of MSCs in Table III, it can be seen that as we change the average power from 600 to $700 \mathrm{~mW}$, there is a change in the sign of the imaginary part of the $b_{n}$ coefficient (which is highlighted by gray color). This is because $\psi_{n}^{\prime}(\beta)$ in the first term of the denominator changes its sign which is responsible for this drastic change. This change in sign is due to the classical resonance responses observed with perfect Lorentzian shapes in high RI medium [37] which is reflected in the force curve, as shown in Fig. 1. In our case, increasing power indirectly increases the RI of the medium, therefore these effects are prominent at high average power. Also, under pulsed excitation, the RI of the particle is significantly higher than the surrounding medium, and such particles cannot be trapped due to enhancement in the scattering force. Consequently, Fano resonance helps in trapping such particles by introducing the concept of negative optical scattering force (NOSF) [39]. Fano resonance occurs at very high average power as we go down in particle size. This is because MSCs are a function of $M \times \alpha$ [Eq. (6)]. Therefore, with a decrease in $\alpha$, to achieve the nonlinear phenomena, the $M$ has to be increased which increases with an increase in average power. The appearance of nonlinear effects helps in trapping high permittivity particles that are difficult to trap under cw excitation. However, no such effects are observed for dipole approximation. Thus, Mie nonparaxial calculation gives us appropriate results as compared to other theories under tight focusing conditions.

For micron-sized particles, the magnitude of $F_{\max }$ and $F_{\min }$ are constant for GO approximation for different particle sizes. In contrast, for EMT, the magnitude of $F_{\max }$ decreases with the increasing particle size, whereas the magnitude of $F_{\text {min }}$ increases slightly with increasing particle size, as discussed in Sec. $8 \mathrm{a}$ in the Appendix. The change in $F_{\min }$ is negligible which can be explained through the force experienced by the particle due to the impulsive action of light when the beam focused on the back surface of the particle (Fig. 6). The focusing of rays or a beam on the back surface of the particle is independent of particle size. Thence, there is no change in the position of reduced coordinate $(z / a)$ for $F_{\min }$ but a change for $F_{\max }$ is observed with increasing particle size for fixed NA. The difference between the value $z / a$ corresponding to $F_{\max }$ for EMT and GO approximation decreases with increasing particle size, as marked by a double sided arrow in Fig. 17 in the Appendix, which indicates that increasing particle size gives good agreement between both the theories under $\mathrm{cw}$ excitation. The noticeable point is that there is no change in magnitude and nature of the force curve for the GO approximation with an increasing particle size which is not feasible because the force acting on any object should change with size of the object. However, in EMT, the magnitude and nature of the force (hump; highlighted by the rectangle in Figs. 17 and 18) curve changes with particle size under cw excitation, as quantitatively listed in Table IV and V in the Appendix.

Under pulsed excitation, an increase in particle size leads to a decrease in $k_{\text {stiffness }}$ which is constant under cw excitation; a detailed discussion is given in Sec. $8 \mathrm{~b}$ in the Appendix. Quite interestingly, the total force exhibits Mie interference behavior near the focus under pulsed excitation due to the significant contribution of nonlinear effects, as shown in Fig. 2. The incorporation of nonlinearity increases the overall RI of the particle, resulting in Mie interference effects due to resonance in MSCs. The periodicity of these oscillations is strongly correlated with Riccati-Bessel functions because 

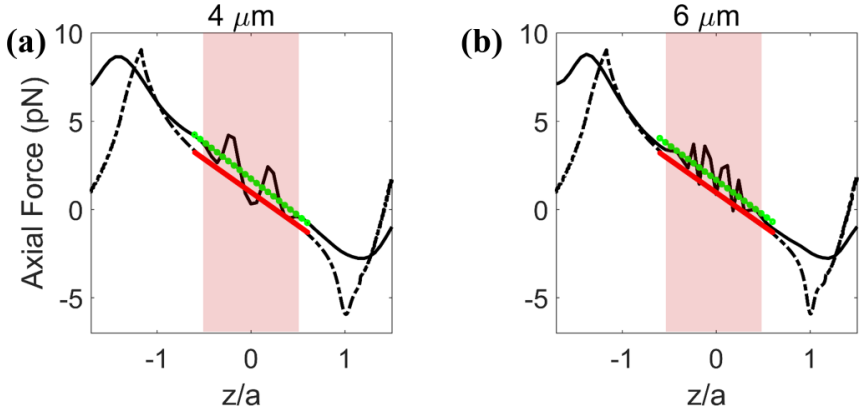

FIG. 2. Plots of trapping force against normalized axial direction $(z / a ; a$ is radius of the particle) for (a) $4-\mu \mathrm{m}$ and (b) $6-\mu \mathrm{m}$ particle radius at $10 \mathrm{~mW}$ average power under pulsed excitations. Solid and dotted curves correspond to EMT and GO approximation, respectively. Red and green curves are linear fits near the focus corresponding to the GO approximation and EMT, respectively.

BSCs [Eq. (7)] are independent on the relative refractive index, average power, and particle size. These oscillations are symmetric along the axial direction because the propagation of Gaussian beam along axial direction is Lorentzian and under pulsed excitation relative refractive index is dependent on the intensity of laser beam profile. This phenomenon is dependent on reflection which is strongly dependent on the particle size. As we go down in particle size, reflection decreases. Therefore, such effects are not present for small-sized particles under similar conditions. If the reflection is high, scattering force can overcome gradient force, and stable trapping of highly scattered particles is difficult. Therefore, to trap high RI particles, a delicate balance between the scattering and gradient force is required by the simultaneous reduction of backscatter force [40] which can be met through Mie structure interference phenomena. The nontrivial interference effects take place between the different transverse magnetic and transverse electric modes which are inherently incorporated through MSCs. Also, the amplitude of Mie interference increases with average power for fixed particle size, as shown in Figs. 19 and 20. This drastic change in the Mie interference can arise due to many nonlinear phenomena such as nonuniform polarization of the particle, dielectric breakdown at high average power [41], Fano resonance [38,39], etc. The salient point is that EMT can capture the occurrence of nonlinear phenomena inside the particle, whereas GO approximation cannot capture such phenomena. Therefore, Mie nonparaxial calculation is much more accurate as compared to other theories.

For the Mie regime, GLMT and EMT differ qualitatively as well as quantitatively, a comparison between different theories is made in Sec. 9 of the Appendix. From the discussion, it is concluded that EMT gives more relevant results in this regime as compared to GLMT. For fixed NA and particle size, $k_{\text {stiffness }}$ of $\mathrm{cw}$ excitation is less than pulsed excitation under similar conditions which implies that pulsed excitation gives better trapping efficiency than cw excitation.

To generalize the studies, we have further analyzed the $F_{\max }$ and $F_{\min }$ using EMT with respect to particle size. $F_{\max }$ initially increases and then decreases [Fig. 3(a)] with increasing particle size whereas $F_{\min }$ continuously decreases [Fig. 3(b)].
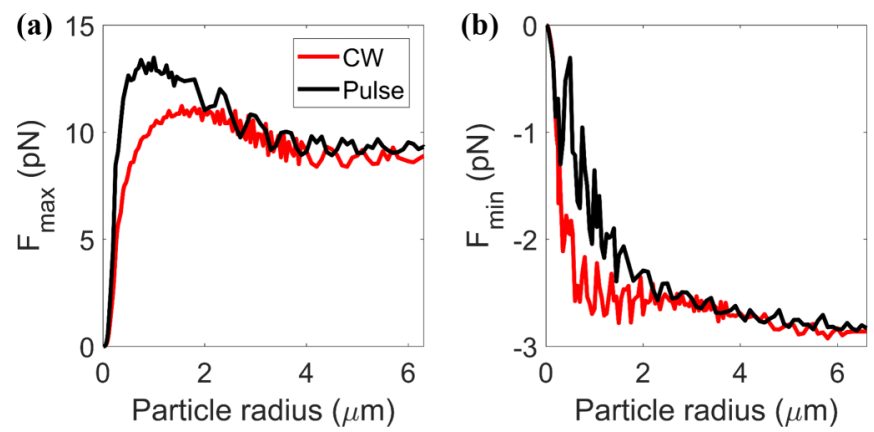

FIG. 3. Plots of (a) $F_{\max }$ (maxima of the force along axial direction) and (b) $F_{\min }$ (minima of the force along axial direction) against particle radius at $10 \mathrm{~mW}$ average power under both $\mathrm{cw}$ and pulsed excitations. $F_{\max }$ and $F_{\min }$ marked by maroon arrow in Fig. 1 .

Under similar conditions, the maximum force experienced by the particle decreases with increasing particle size within the range of micron-sized particles (typically $>4 \mu \mathrm{m}$ ) which was earlier believed to be independent of particle size using GO approximation [5]. Note that no significant difference in $F_{\max }$ and $F_{\min }$ is observed in comparing cw and pulsed excitation for large $(>4 \mu \mathrm{m})$ sized particles. This happens because under pulsed excitation, the appearance of the nonlinear effects significantly modulates the trapping efficiency for nanoparticles. This modulation is even more robust under pulsed excitation as we go towards high average power because the effects of nonlinear phenomena are enhanced significantly.

To study the dynamics of a single particle moving in a three-dimensional (3D) optical trap, phase space trajectories are analyzed. The particle is initially confined and then moved out with the continuous change (magnitude or length) in the velocity vector which is due to the accumulation of energy [20], as shown in Figs. 4(a)-4(c). These phase space trajectories can be directly correlated with the confinement dynamics. With increasing particle size, the equilibrium position shifts towards a positive axial direction, and the confinement region also increases (highlighted by the rectangle). Under pulsed excitation, at $10 \mathrm{~mW}$ average power, a substantial change in magnitude or length of the velocity vector is observed as compared to $\mathrm{cw}$ excitation which indicates the change in momentum for fixed particle size. Hence, it is apparent that the momentum experienced by the particle is higher under pulsed excitation than $\mathrm{cw}$ excitation for $40-\mathrm{nm}$ and $0.4-\mu \mathrm{m}$ particles. For 4- $\mu \mathrm{m}$ particles, change is small compared to $40-\mathrm{nm}$ and $0.4-\mu \mathrm{m}$ particles. Under cw excitation, increasing power does not reflect any change in the trajectory of the particle, but a change in the magnitude of momentum is observed. On the other hand, there is a change in both the trajectory and magnitude of momentum experienced by the particle with increasing average power under pulsed excitation, as discussed in Sec. 10 of the Appendix.

From the above discussion, it can be depicted that under cw excitation, EMT matches with the GO regime and does provide us a reliable prediction of force acting on the particle. However, they fail to agree for the same under the Rayleigh regime. Although, in the literature, it is proposed that GLMT using localized approximation is a general 

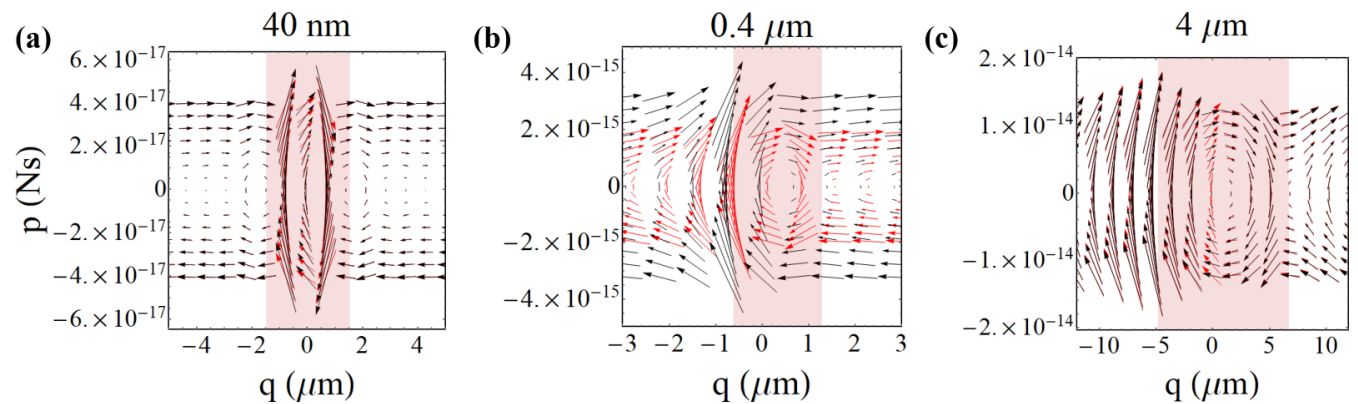

FIG. 4. Plots represent the phase space trajectories of a single particle inside an optical trap for (a) $40 \mathrm{~nm}$, (b) $0.4 \mu \mathrm{m}$, and (c) $4 \mu \mathrm{m}$ particle size at $10 \mathrm{~mW}$ average power under both $\mathrm{cw}$ and pulsed excitations. Red and black vectors correspond to $\mathrm{cw}$ and pulsed excitations, respectively.

theory holding ground for all size limits [34], yet we found that according to the nature of the force curve, it is only applicable for small-sized particles. EMT is reliable in the Mie regime for the force calculations in both $\mathrm{cw}$ and pulsed excitation as compared to GLMT. Thus, overall, EMT gives us more reliable results in all three different regimes.

We conclude that all the different theories are not in good agreement with each other. It is found that the dipole and GLMT approximations do not match with EMT, while GO agrees with EMT under cw excitation. Under pulsed excitation, GO results deviate from EMT at high average power due to nonlinear effects. A change in trapping efficiency is observed for micron-sized particles for changing particle size, which is constant while using GO approximation. For smaller sized particles, pulsed excitation gives better trapping efficiency than cw excitation under similar average power using EMT. Thus, EMT gives more reliable and generalized results as compared to other theories which are reflected from the phase space trajectories as well.

\section{ACKNOWLEDGMENTS}

SERB, DST (Early Career Research Award No. ECR/2016/000467), and IISER Mohali (Start-up Grant and Fellowship to A.D.) are acknowledged for funding.

The authors declare no conflicts of interest.

\section{APPENDIX}

\section{Theory}

\section{a. Dipole approximation}

This theory is applicable for small size particle $(\lambda \gg a)$; where $\lambda$ is the wavelength of the laser beam and $a$ is the radius of the particle. Reduced coordinate force expression for dipole approximation can be written as $[26,42]$

$$
\begin{aligned}
& F^{\text {Dipole }}(z ; r=0) \\
& \quad=F_{\text {scattering }}^{\text {Dipole }}(z ; r=0)+F_{\text {gradient }}^{\text {Dipole }}(z ; r=0), \\
& F_{\text {scattering }}^{\text {Dipole }}(z ; r=0) \\
& \quad=\frac{8 \pi n^{w}(k a)^{4} \mathrm{a}^{2}}{3 c}\left(\frac{M^{2}-1}{M^{2}+2}\right)^{2} I_{\text {peak } / \mathrm{avg}}(z), \\
& F_{\text {gradient }}^{\text {Dipole }}(z ; r=0) \\
& \quad=-\frac{2 \pi n^{w} a^{3}}{c}\left(\frac{M^{2}-1}{M^{2}+2}\right) \frac{8 \tilde{z} /\left(k \omega_{0}^{2}\right)}{1+(2 \tilde{z})^{2}} I_{\text {peak } / \mathrm{avg}}(z),
\end{aligned}
$$

where $\tilde{r}=\frac{r}{\omega_{0}}, \tilde{z}=\frac{z}{k \times \omega_{0}^{2}}, c$ is the speed of light, $k=\frac{2 \pi}{\lambda}$ is the propagating wave vector, $n^{w}$ is the refractive index (RI) of the medium (water), $\omega_{0}=\frac{0.82 \times \lambda}{N A}$ is the spot size at the focal plane where NA is the numerical aperture of the objective lens, $P_{\text {peak/avg }}$ is the peak power for pulsed and average power for continuous-wave (cw) excitation, and $M=\frac{n^{p}}{n^{w}}$ is the relative RI of the particle (polystyrene) to the medium (water). $I_{\text {peak } / \text { avg }}(z)$ is the peak or average intensity of focused Gaussian beam under pulsed or $\mathrm{cw}$ excitation which can be written as $I_{\text {peak/avg }}(z)=\left(\frac{2 P_{\text {peak } / \text { avg }}}{\pi \omega_{0}^{2}}\right) \frac{1}{1+(2 \tilde{z})^{2}}$.

\section{b. GO 3D distribution}

This theory is applicable for large size particles $(\lambda \ll a)$. The GO considering the 3D distribution of light cone force expressions are $[23,24]$

$$
\begin{aligned}
F^{\mathrm{GO}}(z) & =\sum_{\text {cone }} F_{\text {cone, gradient }}(z)+F_{\text {cone, scattering }}(z), \\
F_{\text {cone, gradient }}(z) & =-\frac{n^{w} P_{\text {cone }}}{c}\left(R^{\prime} \sin 2 \theta_{i}-\frac{T^{\prime 2}\left[\sin \left(2 \theta_{i}-2 \theta_{r}\right)+R^{\prime} \sin 2 \theta_{i}\right]}{1+R^{\prime 2}+2 R^{\prime} \cos \left(2 \theta_{r}\right)}\right) \sin \varphi, \\
F_{\text {cone, scattering }}(z) & =\frac{n^{w} P_{\text {cone }}}{c}\left(1+R^{\prime} \cos 2 \theta_{i}-\frac{T^{\prime 2}\left[\cos \left(2 \theta_{i}-2 \theta_{r}\right)+R^{\prime} \cos 2 \theta_{i}\right]}{1+R^{\prime 2}+2 R^{\prime} \cos \left(2 \theta_{r}\right)}\right) \cos \varphi,
\end{aligned}
$$



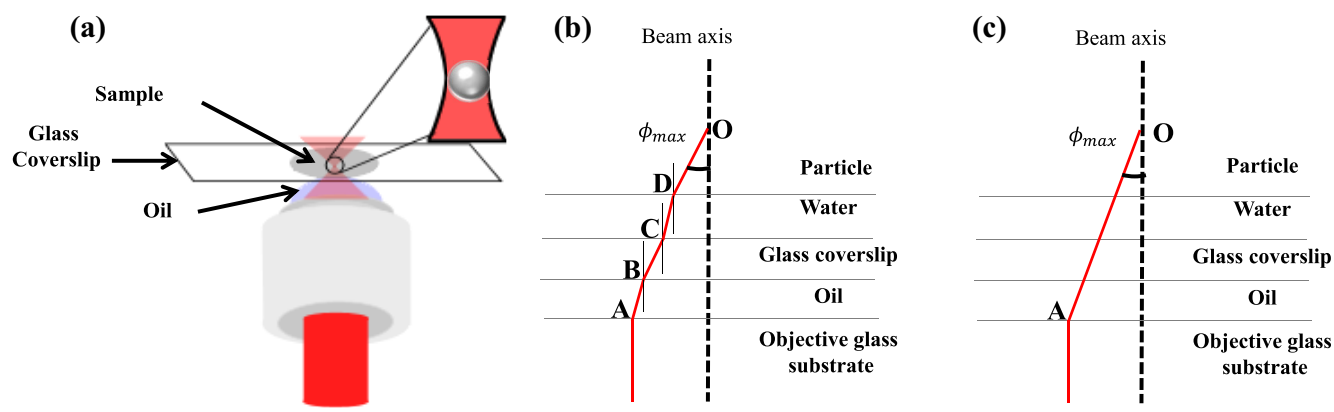

FIG. 5. The schematic diagram (a) of focusing the laser beam onto the sample, ray diagram for (b) old approximation, and (c) new approximation for calculating $\phi_{\max } ; \phi_{\max }$ is the maximum focusing angle with the beam axis.

where $\varphi$ is the angle beyond the objective with beam axis, $R^{\prime}$ and $T$ are the Fresnel reflection and transmission coefficient, and $P_{\text {cone }}=\frac{P_{\text {total }}}{A_{\text {toal }}} \times e^{-2 r^{2} /\left(\omega^{2}+\omega_{0}^{2}\right)} A_{\text {cone }}$ is the power corresponding to a single cone; where $A_{\text {total }}=\pi \omega^{2}+\pi \omega_{0}^{2}$ is the total area under the curve and $A_{\text {cone }}=2 \pi r \delta r+\pi(\delta r)^{2}+\frac{\pi \omega_{0}^{2}}{N}$ is the area of a single light cone, where $r$ is the arc length corresponding to an arbitrary cone whereas $\omega$ is the arc length corresponding to the terminal cone. The force calculations of the large size particles are done for summation over 100 cones [24].

\section{c. GLMT using localized approximation}

This theory is applicable to arbitrary size particles. The generalized Lorentz Mie theory (GLMT) using localized approximation force expressions can be written as [27]

$$
\begin{aligned}
F^{\mathrm{GLMT}}(z)= & F_{\text {scattering }}^{\mathrm{GLMT}}(z)+F_{\text {gradient }}^{\mathrm{GLMT}}(z), \\
F_{\text {scattering }}^{\mathrm{GLMT}}(z)= & \left(\frac{n^{w}}{c}\right)\left(\frac{2 P}{\pi w_{0}^{2}}\right)\left(\frac{\lambda^{2}}{2 \pi}\right) \\
& \times \sum_{n}\left[\left(\frac{2 n+1}{n(n+1)}\right)\left|g_{n}\right|^{2} S_{n, \mathrm{GLMT}}^{(1)}\right], \\
F_{\text {gradient }}^{\mathrm{GLMT}}(z)= & \left(\frac{n^{w}}{c}\right)\left(\frac{2 P}{\pi w_{0}^{2}}\right)\left(\frac{\lambda^{2}}{2 \pi}\right) \\
& \times \sum_{n}\left[\left(\frac{n(n+2)}{n+1}\right) \operatorname{Re}\left[g_{n} g_{n+1}^{*} S_{n, \mathrm{GLMT}}^{(2)}\right]\right]
\end{aligned}
$$
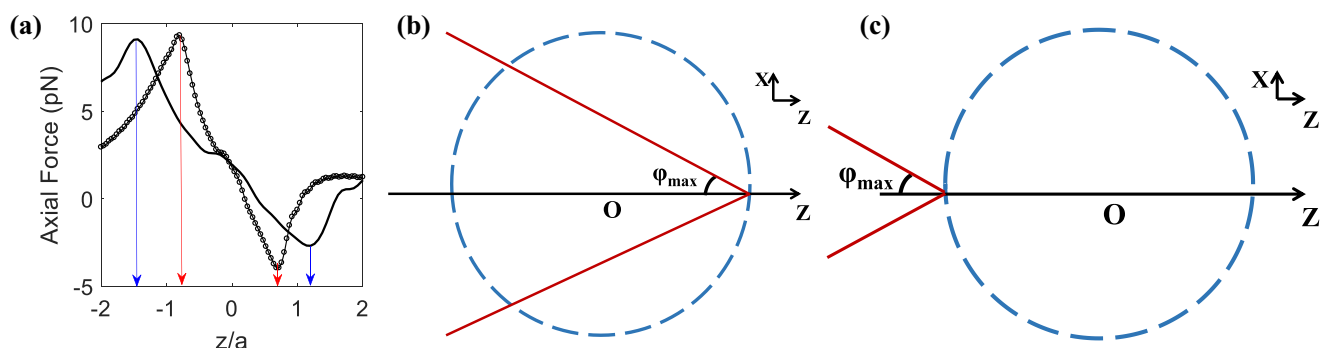

FIG. 6. (a) The plots of trapping force using exact Mie theory for the old and new approximation of $\phi_{\max }$ at $10 \mathrm{~mW}$ average power under cw excitation. Schematic diagram (b) when beam focus at $z / R=-1$ (front surface), and (c) when beam focus at $z / R=1$ (back surface). ficient which can be written as

$$
\begin{gathered}
S_{n, \mathrm{GLMT}}^{(1)}=\operatorname{Re}\left(a_{n}+b_{n}-2 a_{n} b_{n}^{*}\right), \\
S_{n, \mathrm{GLMT}}^{(2)}=\left(a_{n}+b_{n}+a_{n+1}^{*}+b_{n+1}^{*}-2 a_{n} a_{n+1}^{*}-2 b_{n} b_{n+1}^{*}\right),
\end{gathered}
$$

where $g_{n}$ is known as the beam shape coefficient (BSC),

$$
g_{n}=i Q \exp \left[-i Q\left(\frac{\rho_{n}}{\omega_{0}}\right)^{2}\right] \exp \left[i k z_{0}\right],
$$

where $Q=\frac{1}{i+2 \frac{z-z_{0}}{l}}, l=k \omega_{0}^{2}$ is the spreading length, and in our simulations we consider $z_{0}=0$ (on axis) and $\rho_{n}=$ $\left(\frac{n+1 / 2}{2 \pi}\right) \lambda$.

Note that under pulsed excitation, we first calculate the instantaneous force acting on the particle; subsequently, the time-averaged force is calculated by averaging force over one duty cycle [26]:

$$
\begin{gathered}
F_{\mathrm{cw}}=F_{\mathrm{avg}}(\text { under cw excitation }), \\
F_{\text {pulsed }}=\frac{1}{T} \int_{-\tau / 2}^{\tau / 2} F_{\text {peak }} d t \text { (under pulsed excitation) }
\end{gathered}
$$

where $\tau$ is the pulse width, and $T$ is the inverse of the pulse repetition rate. In our simulations, we consider the repetition 

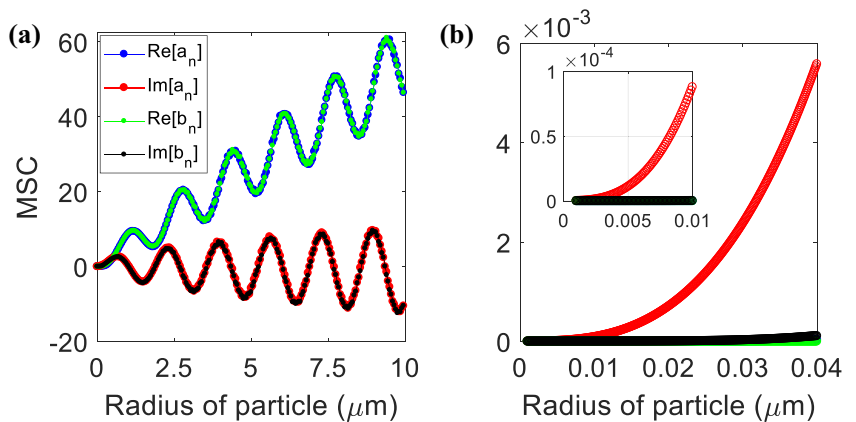

FIG. 7. Plots represent the contribution of Mie scattering coefficients (MSCs) for (a) nanometer to micron sized particles, and (b) zoomed-in regime of nanoparticles (Rayleigh regime only) under cw excitation.

rate high enough $(76 \mathrm{MHz})$ to ignore any artifacts due to particle diffusion.

\section{Parameters}

We choose the central wavelength of the trapping beam to be $800 \mathrm{~nm}$ having Gaussian beam profile under both $\mathrm{cw}$ and pulsed excitation. The $\tau$ and RR are 120 fs and $76 \mathrm{MHz}$, respectively, for a commercially available Ti:sapphire oscillator for which $P_{\text {peak }}=\frac{P_{\text {avg }}}{\operatorname{RRT}} \approx P_{\text {avg }} \times 1.1 \times 10^{5}$. The numerical apertures (NA) for commercial oil-immersion objectives are $\mathrm{NA}=1.4$ and 1.3 (with the typical RI of immersion oil being 1.515); these values are $0.924 \& 0.858$ and $1.228 \& 1.140$ in air and water, respectively. For $800 \mathrm{~nm}$, $\mathrm{A}=0.85$. The linear refractive index of polystyrene and medium (water) is taken as $n_{0}^{p}=1.5782$ and $n_{0}^{w}=1.33$ [26], respectively. The values of nonlinear refractive indices for particle $\left(n_{2}^{p}\right)$ and water $\left(n_{2}^{w}\right)$ are $2.7 \times 10^{-20} \mathrm{~m}^{2} \mathrm{~W}^{-1}$ [43] and $5.9 \times 10^{-17} \mathrm{~m}^{2} \mathrm{~W}^{-1}[44,45]$, respectively.

\section{Calculation for $\phi_{\max }$}

Figure 5 shows the schematic diagram for calculating $\phi_{\max } ; \phi_{\max }$ is the maximum focusing angle with the beam axis. In the literature [33], $\phi_{\max }$ is calculated by using Fig. 5(b). At point $A$, the beam is transmitting from glass substrate (having RI 1.51) to oil (having RI 1.55). In this case, the beam will bend towards the normal depicted by Snell's law. However, at point $B$, the beam will bend away from the normal because the beam is traveling from high to less dense medium. At point $C$, the beam is traveling from glass substrate to water (having RI 1.33), so the beam will bend away from the normal and eventually the beam will focus on the beam axis with $\phi_{\max } \approx \sin ^{-1}\left(\frac{\mathrm{NA}}{n^{w}}\right)$. But this relation of $\phi_{\max }$ does not hold for high NA objective. For example, if we consider $\mathrm{NA}=1.33$ and 1.4 then $\phi_{\max }$ comes out to be $90^{\circ}$ and $90^{\circ}-18.51 i$, which is not possible. To nullify this discrepancy, we assume that these small deviations can be neglected as shown in Fig. 5(c). In other words, the focusing persists throughout the medium and $\phi_{\max }=\sin ^{-1}\left(\frac{\mathrm{NA}}{n_{\mathrm{oil}}}\right)$. The
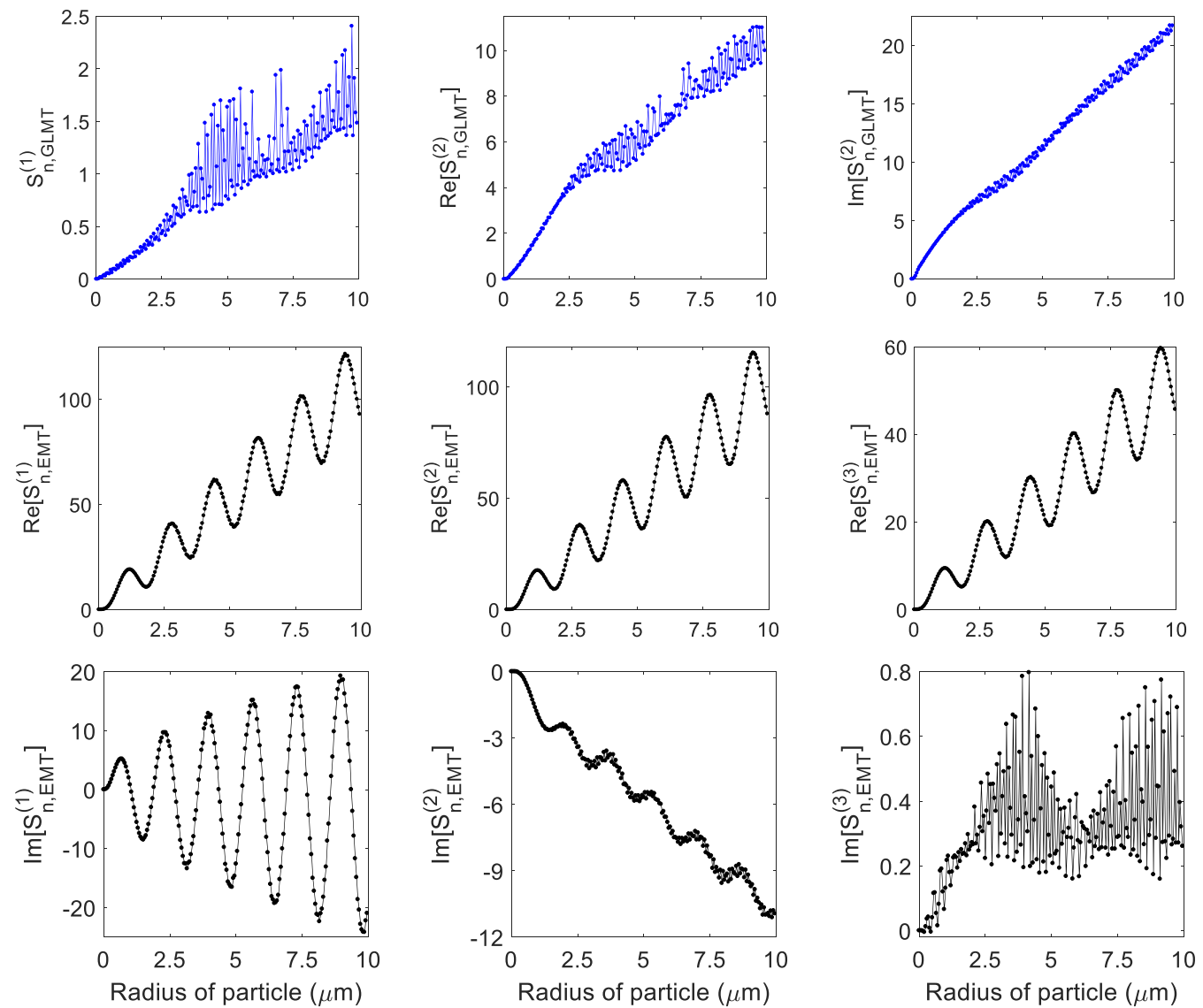

FIG. 8. Plots of scattering coefficients $\left(S_{n, \mathrm{GLMT}}^{(1)}, S_{n, \mathrm{GLMT}}^{(2)}, S_{n, \mathrm{EMT}}^{(1)}, S_{n, \mathrm{EMT}}^{(2)}\right.$, and $\left.S_{n, \mathrm{EMT}}^{(3)}\right)$ against particle size under cw excitation. 

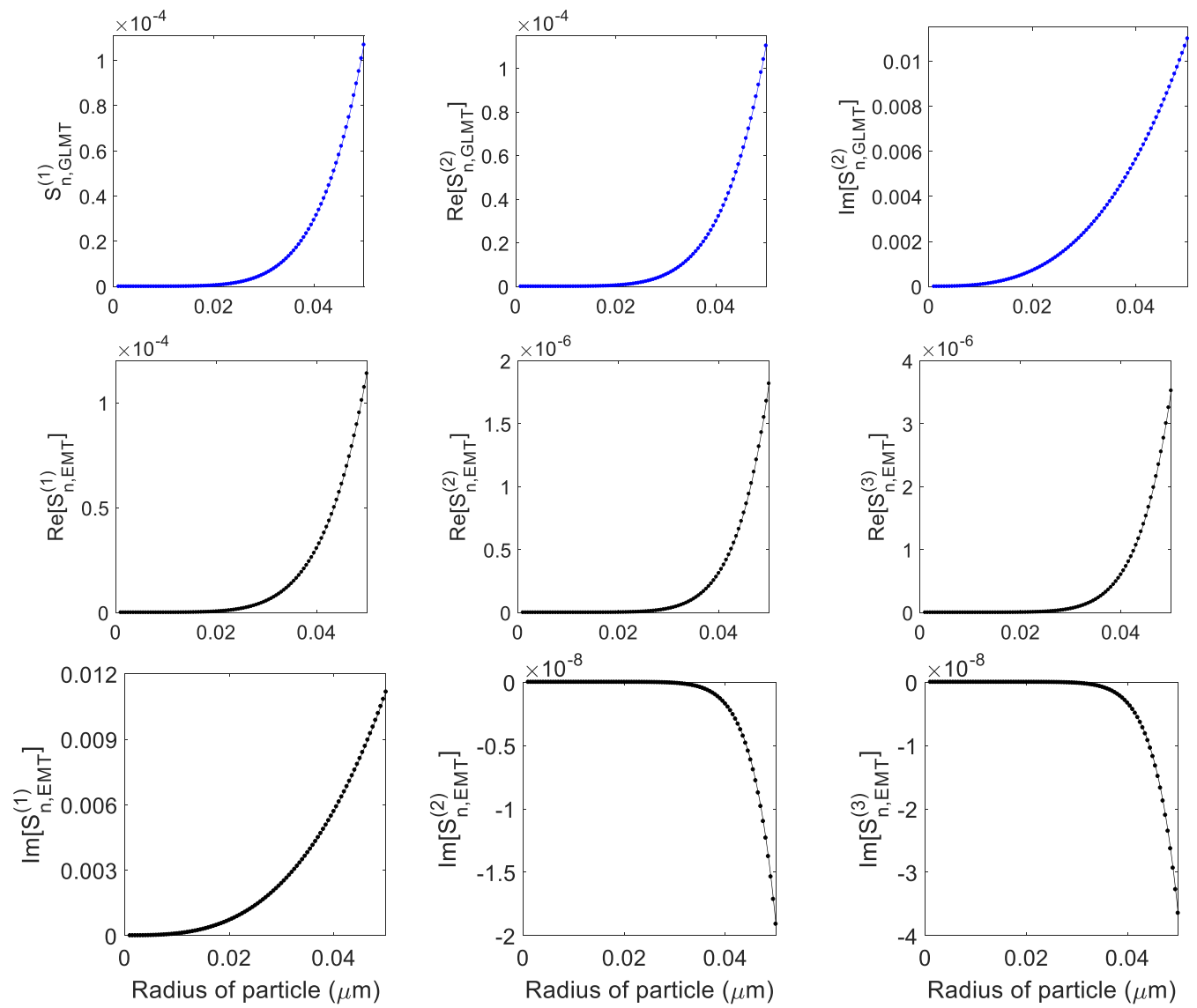

FIG. 9. Plots of scattering coefficients $\left(S_{n, \mathrm{GLMT}}^{(1)}, S_{n, \mathrm{GLMT}}^{(2)}, S_{n, \mathrm{EMT}}^{(1)}, S_{n, \mathrm{EMT}}^{(2)}\right.$, and $\left.S_{n, \mathrm{EMT}}^{(3)}\right)$ against particle size under cw excitation for Rayleigh regime.

redefined approximation gives relevant angles: $59.10^{\circ}$ and $67.53^{\circ}$ for NA 1.3 and 1.4 respectively. Similarly, we can now calculate the force acting on the particle for NA 1.4, unlike the previous definition where the focusing angle cannot be calculated because integrating over a complex angle is not possible. Please note that here we assume that the objective is corrected for spherical aberration when the beam is focused between the glass coverslip and sample chamber. However, we can use Mie-Debye-spherical aberration theory [46,47], for correcting spherical aberration where it is not corrected for the objective under tight focusing conditions.

Please note that while performing the experiment the distance between the coverslip and focus is kept at typically $40-50 \mu \mathrm{m}$ which is sufficiently far from the coverslip glass. Hence, we can safely ignore the generation of evanescent wave. However, this effect is more prominent when we use metal particles instead of dielectric particles.

Figure 6(a) shows the trapping force acting on the 4- $\mu \mathrm{m}$ particle size with $\mathrm{NA}=1.3$ from both the old (circled line) and new approximation (solid line) at $10 \mathrm{~mW}$ average power. It can be seen that a small change in the focusing angle gives a significant change in the trapping force curve. Figure 6(b) shows a schematic diagram when the beam is focusing on the front surface of the particle, and Fig. 6(c) shows the beam focusing on the back surface of the particle. At these points, the scattering force is dominant over the gradient force, which leads to a sharp peak before (back surface) and after (front surface) the boundary. According to old approximation, these peaks lie inside the particle, but this is not possible because when the beam focuses inside, the particle gradient force plays a vital role in the overall force. Please note that according to this convention $z$ is negative so, for negative axial distance, it corresponds to $z / R \leqslant-1$ (the focus is on the front surface of the particle); this is because for $z / R=-1$ the focus is still within the particle so the maximum repelling force exertion must be $z / R<-1$ (and not on the surface of the particle) due to refraction. This is because the bending of light inside the medium might cause the deviation from its actual focus position, so the maximum force acting on the particle must lie on $z / R>1$. The overall conclusion is that when the beam focuses outside the surface (back and front) of the particle, the magnitude of the scattering force is maximizing over the gradient force. This is evident from the sharp peak in the force curve. These positions must be $z / R \leqslant-1$ and $z / R \geqslant 1$. In all the simulations from now on, we use the new approximation of $\phi_{\max }$ to calculate the trapping force using exact Mie theory (EMT).

\section{Scattering coefficients}

\section{a. cw excitation}

Figure 7(a) shows the plots of Mie scattering coefficients (MSCs) against particle size till $10 \mu \mathrm{m}$ for which MSCs summed over 1-150 terms (because all the MSCs converge within this size limit for 150 terms). It can be seen that as per increase in the particle size towards micron-sized particles, 

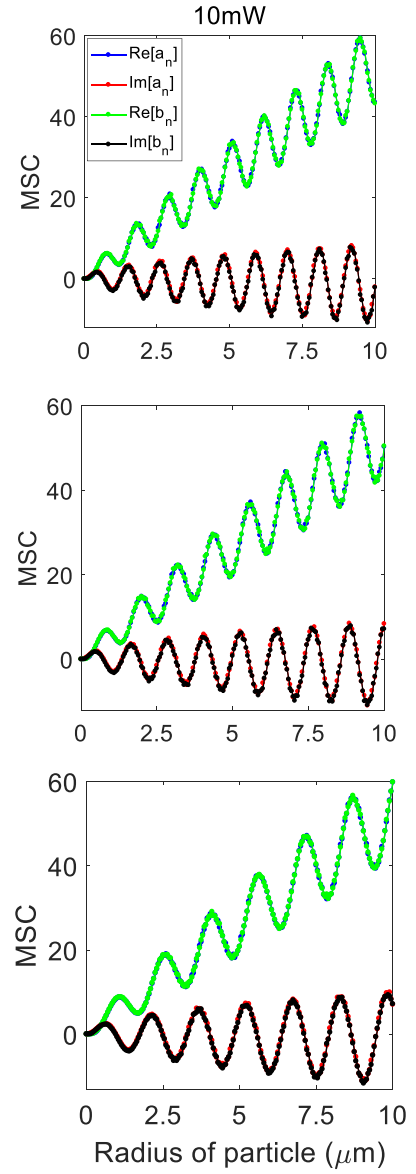
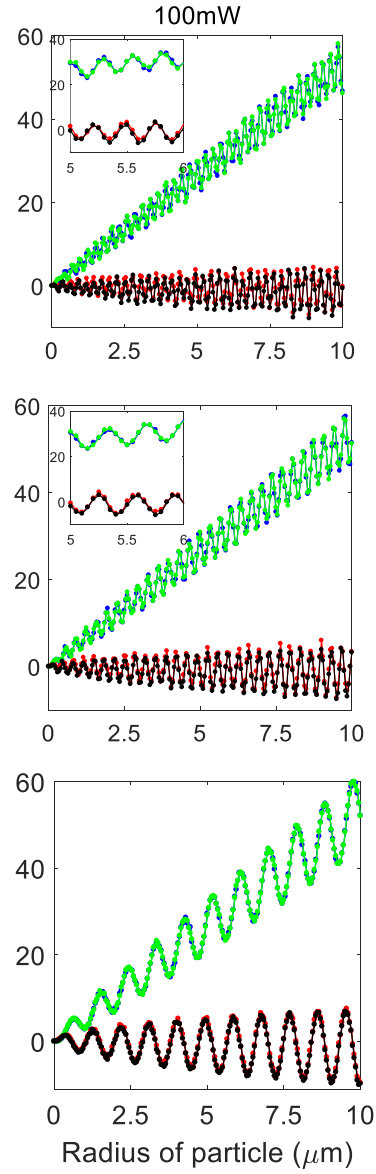
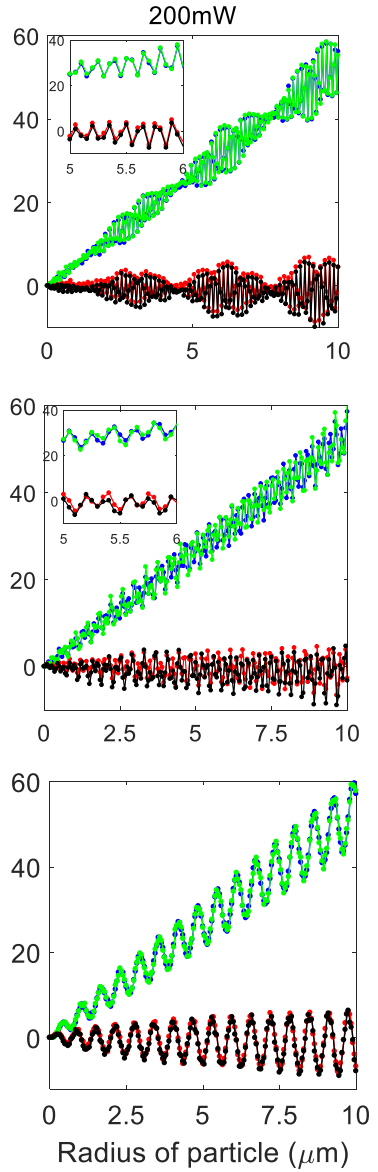

FIG. 10. Plots of MSCs against particle size under pulsed excitation for $z=0 \mu \mathrm{m}$ (focal plane; top panel), $z=1 \mu \mathrm{m}$ (middle panel) and $z=4 \mu \mathrm{m}$ (bottom panel).

all the scattering coefficients $\left(\operatorname{Re}\left[a_{n}\right], \operatorname{Im}\left[a_{n}\right], \operatorname{Re}\left[b_{n}\right]\right.$, and $\left.\operatorname{Im}\left[b_{n}\right]\right)$ are contributing significantly to the force calculation. However, for the nanosized particle [Fig. 7(b)], $\operatorname{Im}\left[a_{n}\right]$ dominates over all other MSCs components, and MSCs converge for summation over 1-10 terms.

Figure 8 shows the plots of scattering coefficients $\left(S_{n, \mathrm{GLMT}}^{(1)}\right.$, $S_{n, \mathrm{GLMT}}^{(2)}, S_{n, \mathrm{EMT}}^{(1)}, S_{n, \mathrm{EMT}}^{(2)}$, and $\left.S_{n, \mathrm{EMT}}^{(3)}\right)$ against particle size for $n$ summed over 1-150 terms. These scattering coefficients contribute significantly to the force calculations for GLMT and EMT. The contribution of the scattering coefficient in GLMT increases with an increase in particle size, but no specific trend has been observed in the curve. In contrast, in the case of EMT, except $\operatorname{Im}\left[S_{n, \mathrm{GLMT}}^{(3)}\right]$, all scattering coefficients follow a trend that shows a Mie interference structure behavior. Figure 9 shows the plots of the scattering coefficient against the particle size for $n$ summed over 1-10 terms in the Rayleigh regime only. For small size particles, the prominent contribution of MSCs is from $\operatorname{Im}\left[S_{n, \mathrm{GLMT}}^{(2)}\right]$ and $\operatorname{Im}\left[S_{n, \mathrm{EMT}}^{(1)}\right]$ in the force calculation. Besides, in both these terms, the dominancy comes from $\operatorname{Im}\left[a_{n}\right]$, as observed in Fig. 7.

\section{b. Pulsed excitation}

Under pulsed excitation, MSCs depend upon axial position (z), NA, $\lambda$, and $P_{\text {peak }}$. Figure 10 shows MSCs for three different planes $(z=0 \mu \mathrm{m}$; focal plane, $1 \mu \mathrm{m}$, and $4 \mu \mathrm{m})$ for fixed NA 1.3 at 10,100 , and $200 \mathrm{~mW}$ average power.
From Fig. 7, it is evident that six maxima appear in the MSCs with particle size up to $10 \mu \mathrm{m}$ under $\mathrm{cw}$ excitation. In contrast, under pulsed excitation, it shows nine maxima for similar conditions (for the focal plane), as can be seen in Fig. 10. From Fig. 10, it is apparent that for a fixed power, the number of maxima decreases as we go away from the focal plane. For example, for a $4-\mu \mathrm{m}$ plane, as we change average power from 10 to $100 \mathrm{~mW}$, the number of maxima is also increased from 6 to 11. Further increase in the average power results in further increases in the maxima and the minima because of the contribution of the nonlinear term (including nonlinearity leads to a change in the RI ratio; here, increasing power means increasing RI of the particle, which corresponds to an increase in the number of oscillations in the Bessel and Hankel function). However, for a 1- $\mu \mathrm{m}$ plane, at $10 \mathrm{~mW}$ average power, there are eight numbers of maxima, but at $100 \mathrm{~mW}$ average power, the periodicity is very small. So, it is tough to say in numbers, and increasing the power afterward gives a wave-form-like pattern. For $z=0 \mu \mathrm{m}$, at $10 \mathrm{~mW}$ average power, there are nine maxima and eight minima. For $100 \mathrm{~mW}$ average power, they are very compact and difficult to count, but interestingly, a further increase in the power leads to the appearance of patches. It is also apparent from Fig. 10 that a small change in the average power leads to a drastic change in the pattern near the focus. However, as we move away from the focus, the pattern changes slowly because the intensity is very high near the focus as compared 

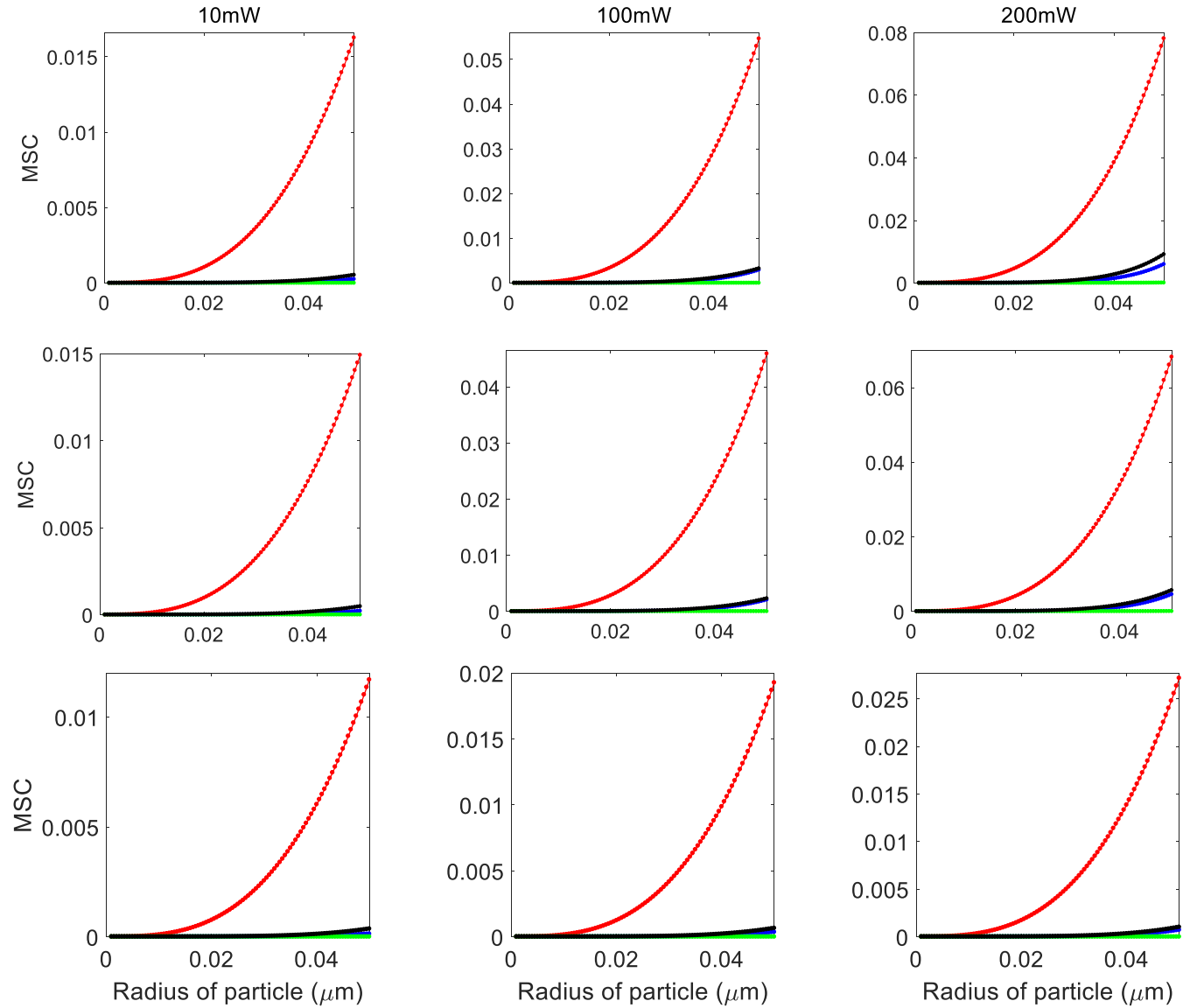

FIG. 11. Plots of MSCs against particle size under pulsed excitation for $z=0 \mu \mathrm{m}$ (focal plane; top panel), $z=1 \mu \mathrm{m}$ (middle panel), and $z=4 \mu \mathrm{m}$ (bottom panel) within Rayleigh regime only.

to a $4-\mu \mathrm{m}$ plane. This behavior is symmetric around the focus and is similar to $\mathrm{cw}$ excitation away from the focus (around $\pm 10 \mu \mathrm{m}$ ).

Figure 11 shows the MSCs against particle size within the Rayleigh limit for three different planes $(z=0 \mu \mathrm{m}$; focal plane, 1 and $4 \mu \mathrm{m}$ ) for fixed NA 1.3 at 10, 100, and $200 \mathrm{~mW}$ average power under pulsed excitation. The nature of MSCs is similar for all three planes at different average power, but the magnitude of the force curve is different. Since MSCs are nonlinearly dependent upon the average power, an increase in average power results in a significant increment in the magnitude of the force curve. However, a substantial decrement in the magnitude of the force curve is observed as we go from the $z=0$ to $4 \mu \mathrm{m}$ plane. For nanoparticles, predominant contribution in the force calculation is from $\operatorname{Im}\left[a_{n}\right]$, and an increase in the average power near the focus increases the magnitude of the force curve and vice versa. Thus, we can say that small change in the axial position $(z)$ and average power leads to a change in the nature of the force curve for both GLMT and EMT.

From the above discussion, we conclude that for nanoparticles, MSCs converge for summed over 1-10 values of $n$. Consequently, force calculations have been done for summation over 1-10 terms of $n$. However, for bigger particle size, MSCs converge for summation over $n$ from 1 to 70,1 to 100 , and 1 to 110 for 4,6 , and $8 \mu \mathrm{m}$, respectively. For the Mie regime, $0.4 \mu \mathrm{m}$ particle size, force calculations converge for summation over 40 terms in both GLMT and EMT.

\section{Beam shaping coefficients (BSCs)}

BSCs are independent of particle properties and average power of the laser beam, and it depends upon, $\phi_{\max }, z$, and summation over the number of terms for the Legendre and exponential function in both EMT and GLMT. BSCs play a crucial role in the force calculations, and these coefficients are the same for both $\mathrm{cw}$ and pulsed excitations. Figure 12 shows the BSCs for summation over ten terms. For smallsized particles, the nature of the force curves is similar to the negative of the imaginary term of the BSCs. The force is calculated by the multiplication of MSCs and BSCs where MSCs are a constant value for fixed particle size and relative RI under $\mathrm{cw}$ excitation. Therefore, the nature of the BSC curve is reflected in the force curve. However, under pulsed excitation, MSCs are also dependent on $z$. Hence, the nature of the force curve is strongly influenced by the nature of BSC curves for cw excitation, while for pulsed excitation, it depends upon both MSCs and BSCs. For large size particles, higher $n$ values also contribute significantly, so summing over more $n(1-150)$ terms leads to an increase in the magnitude of the force while the nature of the force curve remains the same. 

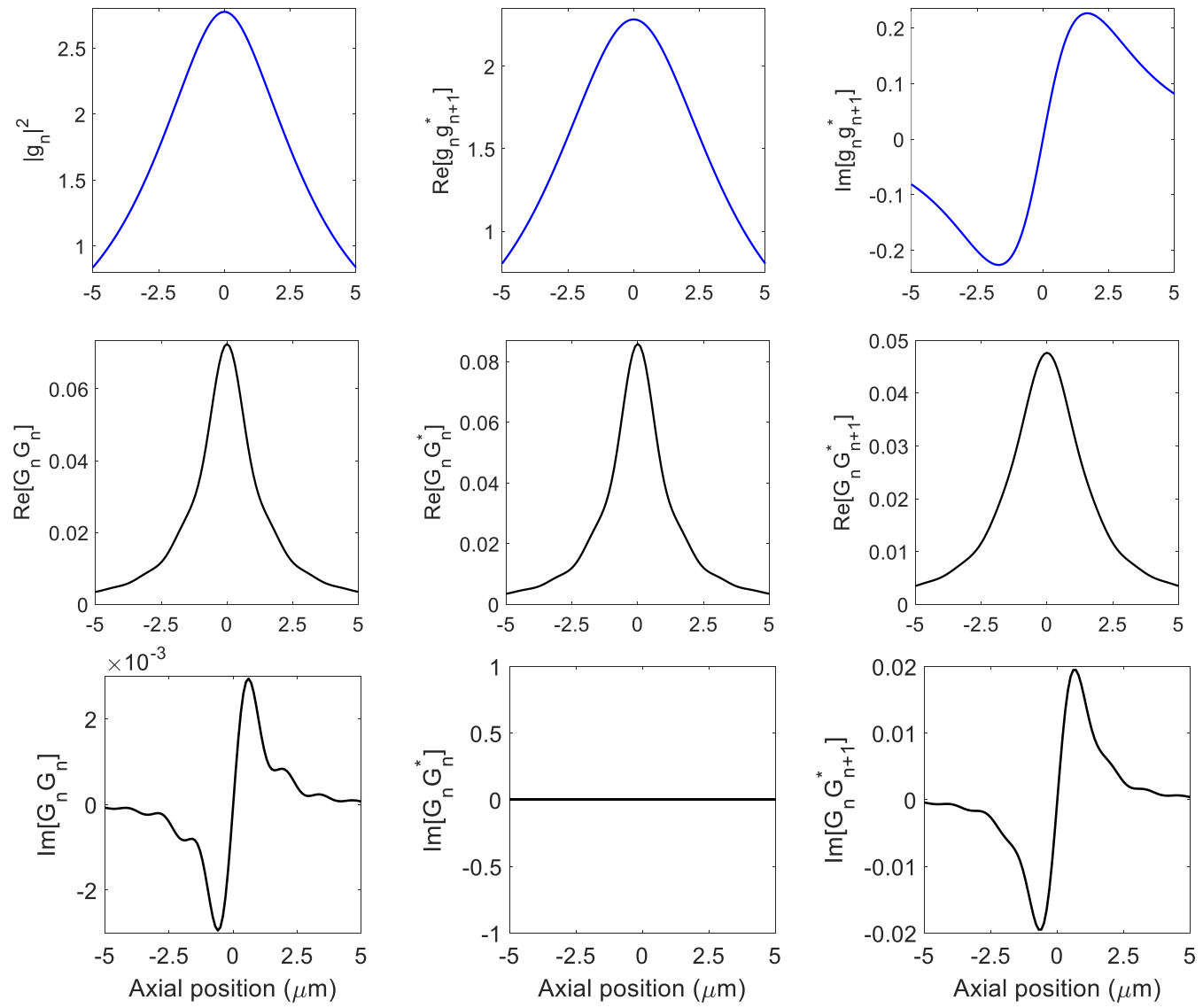

FIG. 12. Plots of beam scattering coefficients (BSCs) against the axial direction for summed over 10 initial terms.

\section{Trap stiffness calculation}

Trap stiffness $\left(\kappa_{\text {stiffness }}\right)$ is calculated by doing a linear fit of force curve near the geometric focus, using $F=-\kappa_{\text {stiffness }} z+$ constant.

\section{Rayleigh regime}

For nanoparticles, the trapping force comparison is done for dipole, GLMT, and EMT. Hereafter, in the Rayleigh regime, the black curve corresponds to the EMT, the blue curve corresponds to the GLMT, and the green curve corresponds to the dipole approximation, unless mentioned otherwise.

\section{a. cw excitation}

All three theories have a significant difference in $F_{\max }, F_{\min }$, and $k_{\text {stiffness. }}$. The force acting on the particle is very confined due to tight focusing conditions in EMT as compared to dipole and GLMT approximations, as shown in Figs. 13(a)-13(c). Therefore, $F_{\max }, F_{\min }$, and $k_{\text {stiffness }}$ is higher for EMT than dipole and GLMT approximations. This is because GLMT and Dipole approximations are solved only by using paraxial approximation, whereas EMT formulation includes tight focusing conditions as well. In addition, an oscillatory nature on the wings of the force curve is observed for EMT owning to BSCs (discussed in Sec. 5). An increase in the particle size leads to an increase in $F_{\max }, F_{\min }$, and $k_{\text {stiffness }}$,

TABLE I. The values of $F_{\max }$ and $F_{\min }$ under cw excitation at $100 \mathrm{~mW}$ average power for EMT, GLMT, and dipole approximation.

\begin{tabular}{|c|c|c|c|c|c|c|c|}
\hline \multirow[b]{2}{*}{ NA } & \multirow[b]{2}{*}{ Particle Size } & \multicolumn{3}{|c|}{$F_{\max }(\mathrm{pN})$} & \multicolumn{3}{|c|}{$F_{\min }(\mathrm{pN})$} \\
\hline & & $20 \mathrm{~nm}$ & $30 \mathrm{~nm}$ & $40 \mathrm{~nm}$ & $20 \mathrm{~nm}$ & $30 \mathrm{~nm}$ & $40 \mathrm{~nm}$ \\
\hline \multirow[t]{3}{*}{1.3} & EMT & 0.0197 & 0.0676 & 0.1044 & -0.0195 & -0.0650 & -0.1504 \\
\hline & GLMT & 0.0030 & 0.0107 & 0.0286 & -0.0028 & -0.0088 & -0.0181 \\
\hline & dipole approx. & 0.0020 & 0.0072 & 0.0193 & -0.0020 & -0.0059 & -0.0118 \\
\hline \multirow[t]{3}{*}{1.4} & EMT & 0.0286 & 0.0975 & 0.2359 & -0.0283 & -0.0946 & -0.2199 \\
\hline & GLMT & 0.0038 & 0.0139 & 0.0365 & -0.0036 & -0.0117 & -0.0246 \\
\hline & dipole approx. & 0.0027 & 0.0096 & 0.0253 & -0.0025 & -0.0080 & -0.0165 \\
\hline
\end{tabular}




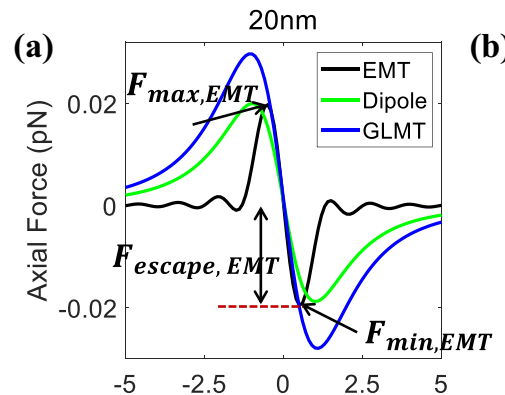

(d)

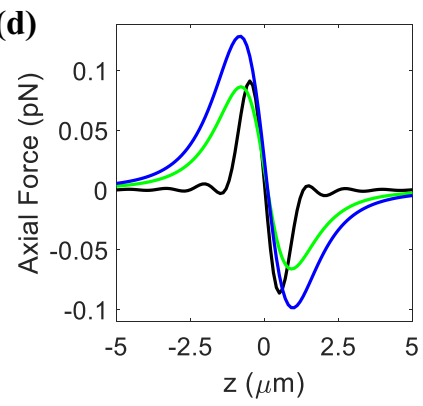

(b)

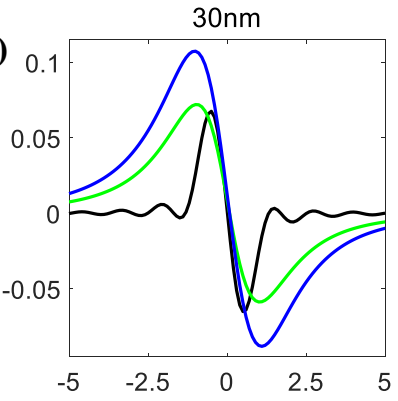

(e)

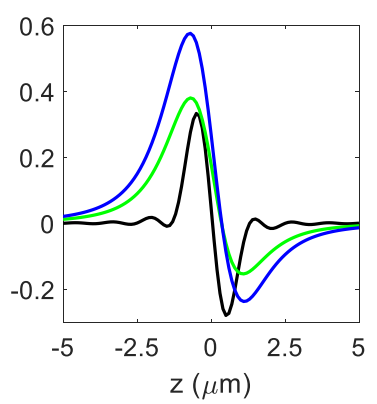

(c)

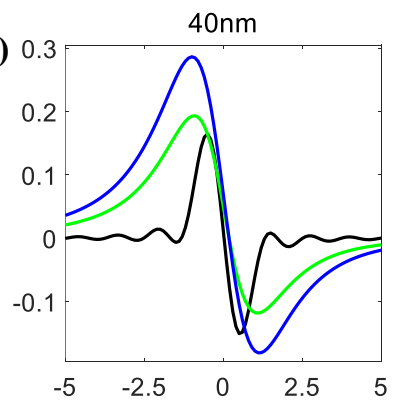

(f)

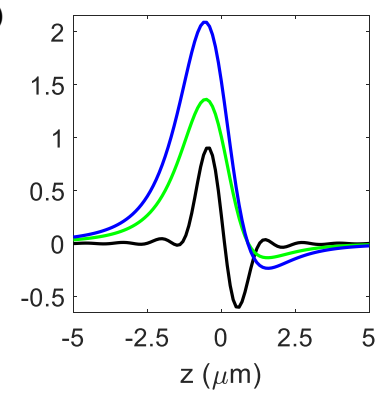

FIG. 13. Plots of trapping force along axial direction using dipole approximation, GLMT, and EMT under both cw (top panel) and pulsed (bottom panel) excitation at $100 \mathrm{~mW}$ average power for fixed NA $=1.3$. Just for clear visualization, we multiplied the dipole and GLMT approximations force curves by a factor of 10 .

and consequently increases the difference between theories. For small size particles (a few nanometers), the trapping force is symmetric (the magnitude of $F_{\max }$ and $F_{\min }$ is the same) about the axis. However, an increase in particle size results in an asymmetric force (the magnitude of $F_{\max }$ and $F_{\min }$ is not the same) due to the significant contribution of scattering force under similar conditions which can be seen from Table I. Also, a significant change in the magnitude of the force curve is observed by increasing NA, as shown in Figs. 14(a)-14(c). Under cw excitation, increasing particle size increases the magnitude of $F_{\text {escape }}$ [as marked in Fig. 13(a)] whereas $F_{\text {escape }}$ decreases with increasing particle size under pulsed excitation due to significant enhancement in scattering force [26].

\section{b. Pulsed excitation}

Under pulsed excitation, an enhancement in the magnitude of $F_{\max }, F_{\min }$, and $k_{\text {stiffness }}$ is observed as compared to cw excitation for EMT, GLMT, and dipole approximation, as shown in Figs. 13(d)-13(f). Including nonlinearity leads to more
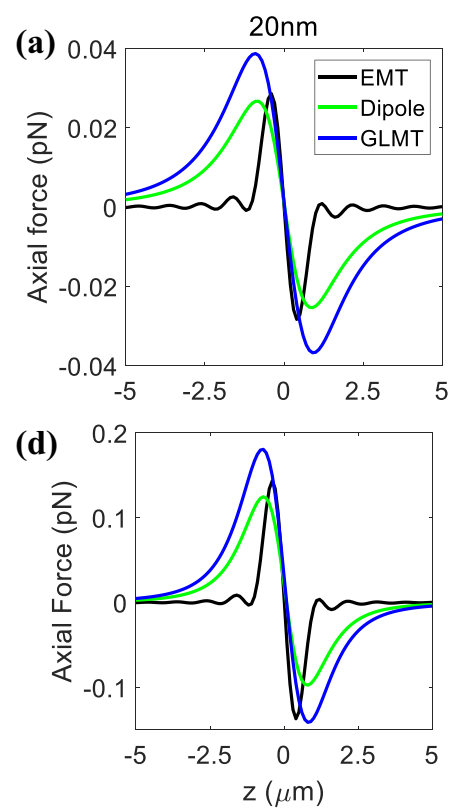

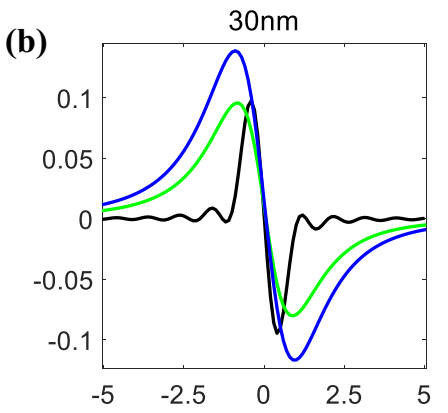

(e)

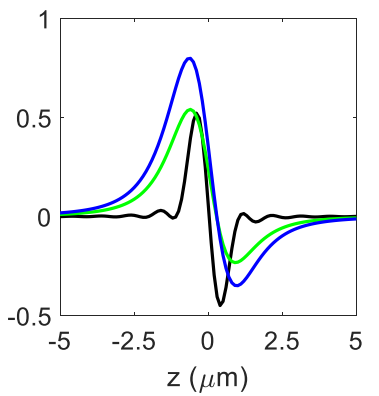

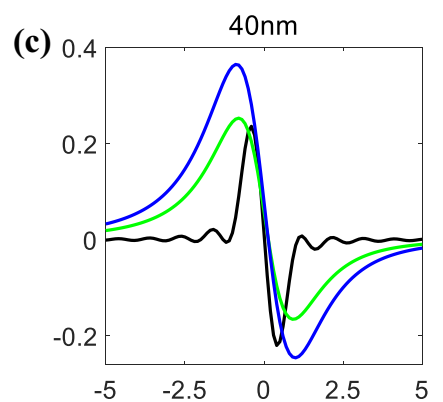

(f)

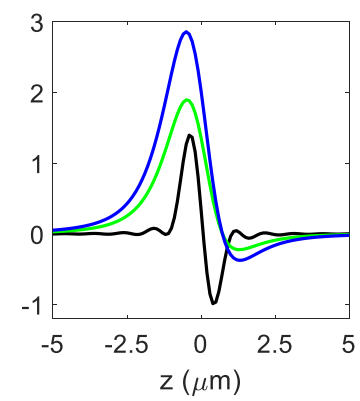

FIG. 14. Plots of trapping force along axial direction for dipole approximation, GLMT, and EMT under both cw (top panel) and pulsed (bottom panel) excitation at $100 \mathrm{~mW}$ average power for fixed NA = 1.4. Just for clear visualization, we multiplied the dipole and GLMT approximations force curves by a factor of 10 . 
TABLE II. The values of $F_{\max }$ and $F_{\min }$ under pulsed excitation at $100 \mathrm{~mW}$ average power for EMT, GLMT, and dipole approximation.

\begin{tabular}{|c|c|c|c|c|c|c|c|}
\hline \multirow[b]{2}{*}{ NA } & \multirow[b]{2}{*}{ Particle Size } & \multicolumn{3}{|c|}{$F_{\max }(\mathrm{pN})$} & \multicolumn{3}{|c|}{$F_{\min }(\mathrm{pN})$} \\
\hline & & $20 \mathrm{~nm}$ & $30 \mathrm{~nm}$ & $40 \mathrm{~nm}$ & $20 \mathrm{~nm}$ & $30 \mathrm{~nm}$ & $40 \mathrm{~nm}$ \\
\hline \multirow[t]{3}{*}{1.3} & EMT & 0.0910 & 0.3330 & 0.9011 & -0.0863 & -0.2795 & -0.6009 \\
\hline & GLMT & 0.0128 & 0.0576 & 0.2089 & -0.0098 & -0.0237 & -0.0234 \\
\hline & dipole approx. & 0.0086 & 0.0380 & 0.1361 & -0.0066 & -0.0154 & -0.0134 \\
\hline \multirow[t]{3}{*}{1.4} & EMT & 0.1433 & 0.5215 & 1.3961 & -0.1370 & -0.4492 & -0.9858 \\
\hline & GLMT & 0.0180 & 0.0798 & 0.2859 & -0.0141 & -0.0349 & -0.0374 \\
\hline & dipole approx. & 0.0125 & 0.0541 & 0.1896 & -0.0097 & -0.0233 & -0.0223 \\
\hline
\end{tabular}

asymmetry in the nature of the force curve as compared to $\mathrm{cw}$ excitation for a fixed NA and particle size for all three theories which can be seen quantitatively by comparing Tables I and II. Also, a significant change in the force curve is observed by increasing NA and particle size, as shown in Figs. 14(d)-14(f). Under pulsed excitation, force is not directly proportional to the average power, therefore, the magnitude of $F_{\max }, F_{\min }$, and $k_{\text {stiffness }}$ increases nonlinearly with increasing average power. The contribution of nonlinearity changes the nature of the force curve enormously at $100 \mathrm{~mW}$ average power, and this change is even more prominent with increasing average power because the contribution of nonlinearity increases. Escape force $\left[F_{\text {escape }}\right.$, marked by a double-sided arrow in Fig. 13(a)] vanishes for both dipole and GLMT approximations at 600 $\mathrm{mW}$ average power [Fig. 1(a) in the main text] which implies that potential becomes unbound, and particles cannot be trapped. Under similar conditions, EMT shows a considerable $F_{\text {escape }}$ which indicates confined trapping. However, an increase in the average power exhibits a drastic change in the nature of the force curve by rising spikes near the focus for EMT, as shown in Fig. 1(b) in the main text. The corresponding values of MSCs are listed in Table III. These spikes appear at high average power owning to the contribution of nonlinear effects, and the origin of these spikes in the force curve is from MSCs. For fixed particle size, at high NA, these effects occur at low average power as compared to lower NA because nonlinear effects increase remarkably due to tight focusing conditions. At $600 \mathrm{~mW}$ average power, there is no oscillation

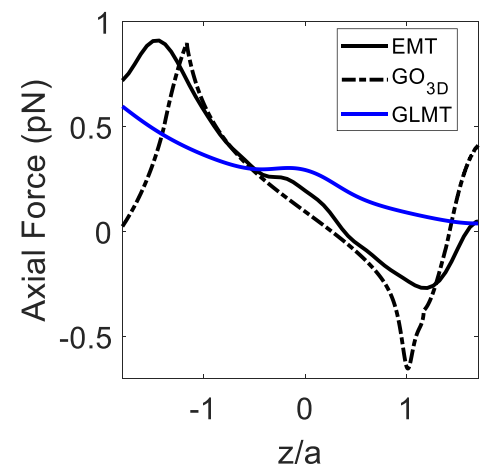

FIG. 15. Plots of trapping force against normalized axial direction using GO 3D distribution, GLMT, and EMT for $4 \mu \mathrm{m}$ particle size at $1 \mathrm{~mW}$ average power under $\mathrm{cw}$ excitation for fixed NA 1.3. or spikes in the case of EMT but they are present in GLMT because the contribution of BSCs overcome this nonlinear phenomenon in EMT.

\section{Geometric optics (GO) regime}

For micron-sized particles, the trapping force comparison is done for EMT, GO 3D distribution, and GLMT. Hereafter in the GO regime, the black curve corresponds to the EMT, the black dash curve corresponds to the GO 3D distribution, and the blue curve corresponds to the GLMT unless mentioned otherwise. Red and green curves are linear fits near the focus corresponding to the GO 3D distribution and EMT, respectively.

\section{a. cw excitation}

Figure 15 depicts that the trapping force curves for EMT and GO 3D distribution are comparable, but GLMT is not. Locally, the nature of force near the focus using GLMT is qualitatively in agreement with EMT. However, the order of magnitude and symmetricity about the axis is very different from EMT and GO 3D distribution. An individual contribution of scattering and gradient force for GLMT can be seen from Fig. 16. No splitting is observed in the scattering force, as seen in GO 3D distribution. The salient point here is that near the focus, a hump is present in both GLMT and EMT because of MSCs. However, no such local distortion is present in the GO 3D distribution curve. It is evident that GLMT

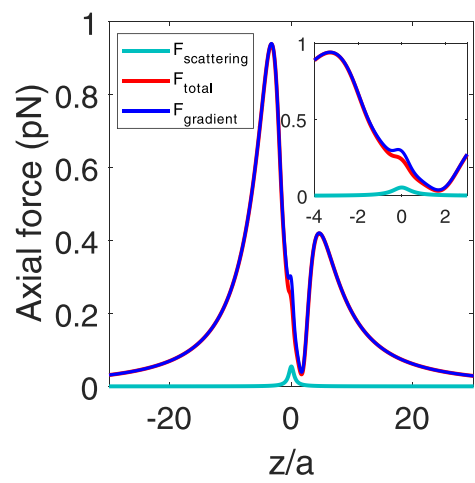

FIG. 16. Plots of trapping force against normalized axial direction using GLMT approximation for $4 \mu \mathrm{m}$ particle with fixed NA 1.3 at $1 \mathrm{~mW}$ average power under $\mathrm{cw}$ excitation. 
TABLE III. The value of MSCs for different axial position $(z)$ at different average power under pulsed excitation for summation over $n=1-10$.

\begin{tabular}{lcrrr}
\hline \hline MSCs & $z(\mu \mathrm{m})$ & $500 \mathrm{~mW}$ & $600 \mathrm{~mW}$ & $700 \mathrm{~mW}$ \\
\hline$a_{n}$ & 0 & $0.0025+i 0.050$ & $0.00262+i 0.0515$ & $0.0028+i 0.053$ \\
& 0.4 & $0.0024+i 0.049$ & $0.00257+i 0.0510$ & $0.0027+i 0.052$ \\
$b_{n}$ & 0 & $5.47 \times 10^{-4}+i 0.023$ & $5.5 \times 10^{-3}+i 0.074$ & $0.024-i 0.154$ \\
& 0.4 & $3.6 \times 10^{-4}+i 0.019$ & $2.2 \times 10^{-3}+i 0.047$ & $0.996-i 0.066$ \\
\hline \hline
\end{tabular}

TABLE IV. The values of $F_{\max }$ under cw excitation at $1 \mathrm{~mW}$ average power for GO 3D distribution and EMT.

\begin{tabular}{|c|c|c|c|c|c|c|c|}
\hline \multicolumn{2}{|c|}{ Particle size } & \multicolumn{2}{|c|}{$4 \mu \mathrm{m}$} & \multicolumn{2}{|c|}{$6 \mu \mathrm{m}$} & \multicolumn{2}{|c|}{$8 \mu \mathrm{m}$} \\
\hline Numerical aperture & Method (quantity) & GO 3D distribution & EMT & GO 3D distribution & EMT & GO 3D distribution & EMT \\
\hline 1.3 & force $(\mathrm{pN})$ & 0.90 & 0.91 & 0.90 & 0.86 & 0.90 & 0.83 \\
\hline 1.4 & force $(\mathrm{pN})$ & 0.97 & 0.93 & 0.96 & 0.88 & 0.96 & 0.85 \\
\hline
\end{tabular}

TABLE V. The values of $F_{\min }$ under cw excitation at $1 \mathrm{~mW}$ average power for GO 3D distribution and EMT.

\begin{tabular}{|c|c|c|c|c|c|c|c|}
\hline \multicolumn{2}{|c|}{ Particle size } & \multicolumn{2}{|l|}{$4 \mu \mathrm{m}$} & \multicolumn{2}{|l|}{$6 \mu \mathrm{m}$} & \multicolumn{2}{|l|}{$8 \mu \mathrm{m}$} \\
\hline Numerical aperture & Method (quantity) & GO 3D distribution & EMT & GO 3D distribution & EMT & GO 3D distribution & EMT \\
\hline 1.3 & force $(\mathrm{pN})$ & -0.65 & -0.27 & -0.65 & -0.28 & -0.65 & -0.28 \\
\hline 1.4 & force $(\mathrm{pN})$ & -0.91 & -0.33 & -0.91 & -0.34 & -0.91 & -0.34 \\
\hline
\end{tabular}

TABLE VI. The values of $k_{\text {Stiffness }}$ under $\mathrm{cw}$ excitation at $1 \mathrm{~mW}$ average power for GO 3D distribution and EMT.

\begin{tabular}{|c|c|c|c|c|c|c|c|}
\hline \multicolumn{2}{|c|}{ Particle size } & \multicolumn{2}{|l|}{$4 \mu \mathrm{m}$} & \multicolumn{2}{|l|}{$6 \mu \mathrm{m}$} & \multicolumn{2}{|l|}{$8 \mu \mathrm{m}$} \\
\hline Numerical aperture & Method (quantity) & GO 3D distribution & EMT & GO 3D distribution & EMT & GO 3D distribution & EMT \\
\hline 1.3 & $k_{\text {stiffness }}\left(\frac{\mathrm{pN}}{\mu \mathrm{m}}\right)$ & 0.38 & 0.35 & 0.38 & 0.35 & 0.37 & 0.35 \\
\hline 1.4 & & 0.47 & 0.41 & 0.47 & 0.41 & 0.47 & 0.41 \\
\hline
\end{tabular}

TABLE VII. The values of $F_{\max }$ under pulsed excitation at $1 \mathrm{~mW}$ average power for GO 3D distribution and EMT.

\begin{tabular}{|c|c|c|c|c|c|c|c|}
\hline \multicolumn{2}{|c|}{ Particle size } & \multicolumn{2}{|l|}{$4 \mu \mathrm{m}$} & \multicolumn{2}{|l|}{$6 \mu \mathrm{m}$} & \multicolumn{2}{|l|}{$8 \mu \mathrm{m}$} \\
\hline Numerical aperture & Method (quantity) & GO 3D distribution & EMT & GO 3D distribution & EMT & GO 3D distribution & EMT \\
\hline 1.3 & force $(\mathrm{pN})$ & 0.90 & 0.90 & 0.90 & 0.87 & 0.90 & 0.83 \\
\hline 1.4 & force $(\mathrm{pN})$ & 0.97 & 0.92 & 0.97 & 0.89 & 0.97 & 0.85 \\
\hline
\end{tabular}

TABLE VIII. The values of $F_{\min }$ under pulsed excitation at $1 \mathrm{~mW}$ average power for GO 3D distribution and EMT.

\begin{tabular}{|c|c|c|c|c|c|c|c|}
\hline \multicolumn{2}{|c|}{ Particle size } & \multicolumn{2}{|l|}{$4 \mu \mathrm{m}$} & \multicolumn{2}{|l|}{$6 \mu \mathrm{m}$} & \multicolumn{2}{|l|}{$8 \mu \mathrm{m}$} \\
\hline Numerical aperture & Method (quantity) & GO 3D distribution & EMT & GO 3D distribution & EMT & GO 3D distribution & EMT \\
\hline 1.3 & force $(\mathrm{pN})$ & -0.64 & -0.27 & -0.64 & -0.28 & -0.64 & -0.28 \\
\hline 1.4 & force $(\mathrm{pN})$ & -0.91 & -0.33 & -0.91 & -0.34 & -0.91 & -0.34 \\
\hline
\end{tabular}

TABLE IX. The values of $k_{\text {stiffness }}$ under pulsed excitation at $1 \mathrm{~mW}$ average power for GO 3D distribution and EMT.

\begin{tabular}{|c|c|c|c|c|c|c|c|}
\hline \multicolumn{2}{|c|}{ Particle size } & \multicolumn{2}{|l|}{$4 \mu \mathrm{m}$} & \multicolumn{2}{|l|}{$6 \mu \mathrm{m}$} & \multicolumn{2}{|l|}{$8 \mu \mathrm{m}$} \\
\hline Numerical aperture & Method (quantity) & GO 3D distribution & EMT & GO 3D distribution & EMT & GO 3D distribution & EMT \\
\hline 1.3 & $k_{\text {stiffnes }}\left(\frac{\mathrm{pN}}{\mu \mathrm{m}}\right)$ & 0.38 & 0.36 & 0.38 & 0.36 & 0.37 & 0.36 \\
\hline 1.4 & & 0.47 & 0.41 & 0.47 & 0.41 & 0.47 & 0.42 \\
\hline
\end{tabular}



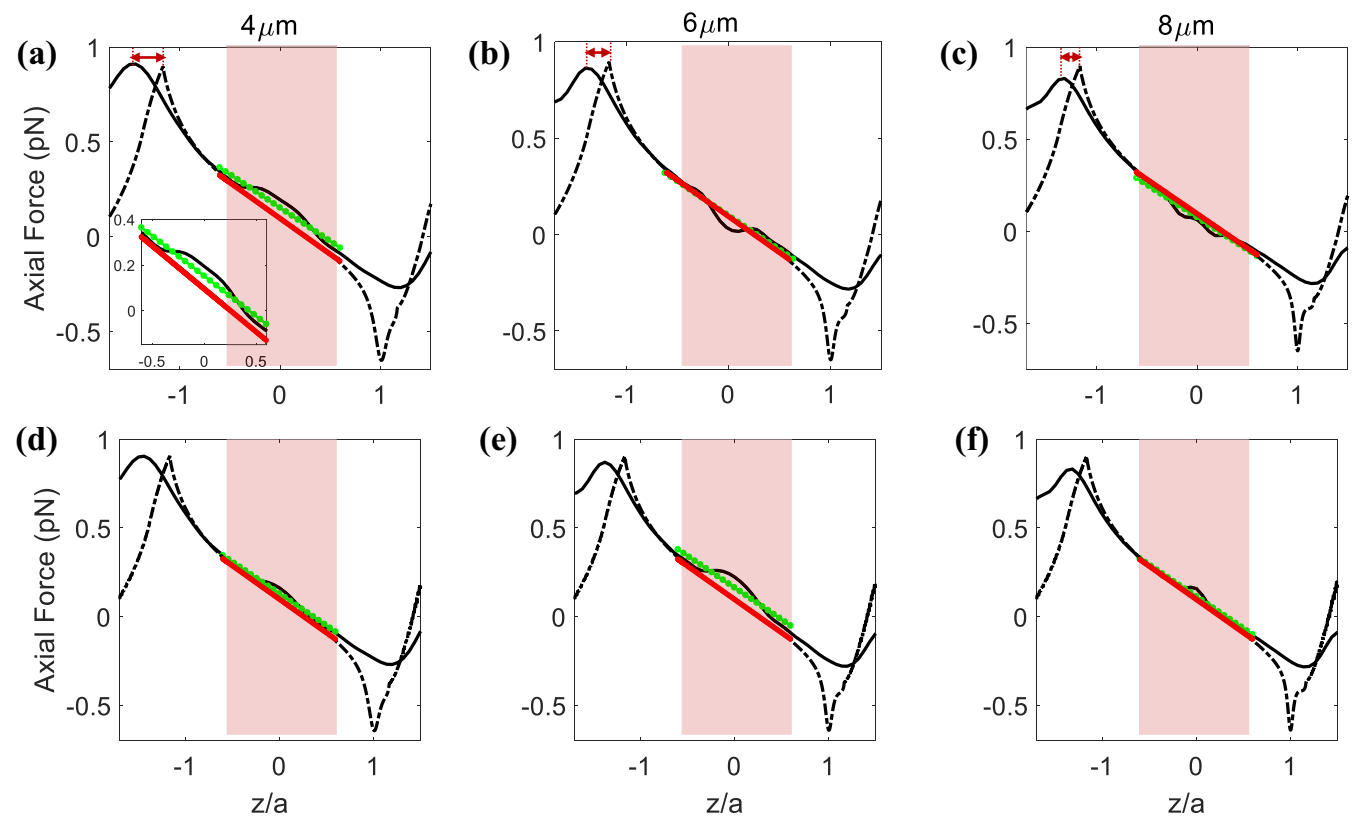

FIG. 17. Plots of trapping force against normalized axial direction using GO 3D distribution and EMT under both cw (top panel) and pulsed (bottom panel) excitation at $1 \mathrm{~mW}$ average power for fixed NA $=1.3$. Highlighted regime indicates the change in the nature of curve near the focus and double sided maroon arrow indicates the difference in the peak positions with increasing particle size.

using localized approximation is not a good approximation to calculate the force acting on the particle for large size particles. Consequently, in further analysis, the comparison is made for EMT and GO 3D distribution for the GO regime.

From Figs. 17(a)-17(c), it is observed that the magnitude of $F_{\max }$ and $F_{\min }$ are constant for GO 3D distribution for different particle sizes, as quantitatively listed in Tables IV and V. In contrast, for EMT, the magnitude of $F_{\max }$ decreases with increasing particle size whereas the magnitude of $F_{\text {min }}$ increases slightly with increasing particle size. For further analysis, we also calculated $k_{\text {stiffness }}$ (Table VI) to get an appropriate analogy. Interestingly, no change in $k_{\text {stiffness }}$ is observed with increasing particle size for both theories, however, there is a significant change in $k_{\text {stiffness }}$ for $\mathrm{NA}=1.4$, as compared to $\mathrm{NA}=1.3$, that can be seen from Figs. 18(a)-18(c). The difference between the axial position $(z)$ of $F_{\max }$ for EMT and GO 3D distribution decreases with increasing particle size [marked by the double-sided arrow in Figs. 17(a)-17(c)]. Thus, increasing particle size gives good agreement between both theories under cw excitation. Consequently, we can say that EMT gives relevant results as compared to GO 3D distribution.

\section{b. Pulsed excitation}

Under pulsed excitation, a qualitative change in the hump (highlighted by the pink rectangle) is observed as compared to cw excitation, as shown in Figs. 17(d)-17(f). In terms of the magnitude of $F_{\max }$ and $F_{\min }$, no significant change is observed, whereas a change in the nature of the force curve near the focus is observed, which is highlighted by the pink rectangle. This change appears due to the contribution of nonlinearity under pulsed excitation. On comparing NA of 1.3 and 1.4, trap stiffness is more for NA 1.4 because increasing NA gives more confinement due to a tight focusing condition, listed in Table VI and shown in Figs. 18(d)-18(f). Also, for fixed particle size, increasing NA increases the magnitude of $F_{\text {max }}$ and $F_{\text {min }}$ due to tight focusing. Figure 20 shows the trapping force

TABLE X. The values of $k_{\text {stiffness }}$ under pulsed excitation at different average power for GO 3D distribution and EMT.

\begin{tabular}{|c|c|c|c|c|c|c|c|}
\hline \multicolumn{2}{|c|}{ Particle size } & \multicolumn{2}{|l|}{$4 \mu \mathrm{m}$} & \multicolumn{2}{|l|}{$6 \mu \mathrm{m}$} & \multicolumn{2}{|l|}{$8 \mu \mathrm{m}$} \\
\hline Numerical aperture & Power $(\mathrm{mW})$ & GO 3D distribution & EMT & GO 3D distribution & EMT & GO 3D distribution & EMT \\
\hline \multirow[t]{3}{*}{1.3} & 1 & 0.38 & 0.36 & 0.38 & 0.36 & 0.37 & 0.36 \\
\hline & 10 & 3.77 & 4.16 & 3.75 & 3.94 & 3.74 & 3.81 \\
\hline & 100 & 36.78 & - & 37.15 & - & 37.24 & - \\
\hline \multirow[t]{3}{*}{1.4} & 1 & 0.47 & 0.41 & 0.47 & 0.41 & 0.47 & 0.42 \\
\hline & 10 & 4.69 & 4.87 & 4.66 & 4.56 & 4.65 & 4.44 \\
\hline & 100 & 46.77 & - & 46.63 & - & 46.55 & - \\
\hline
\end{tabular}




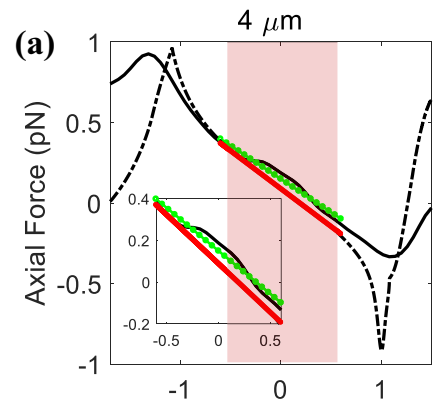

(b)

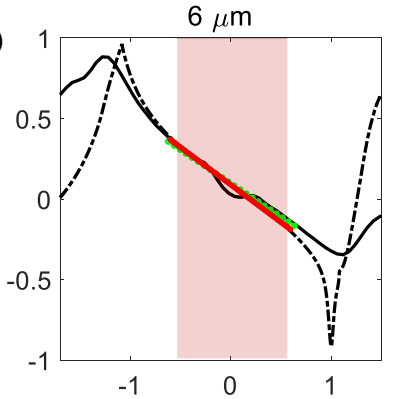

(d)

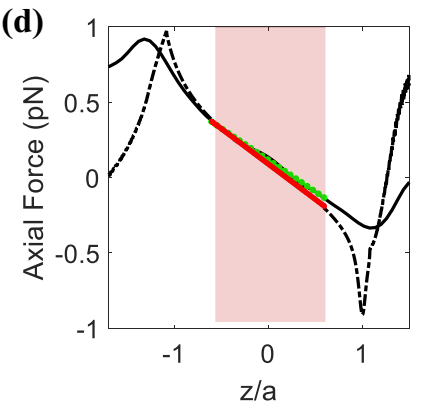

(e)

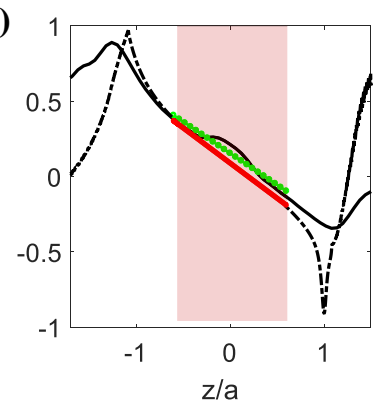

(c)

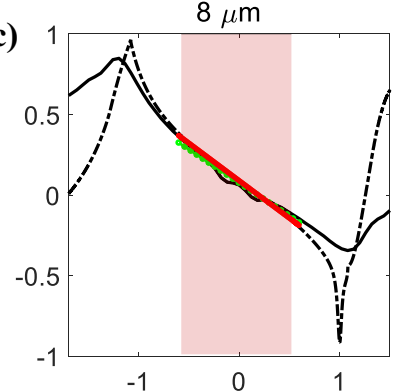

(f)

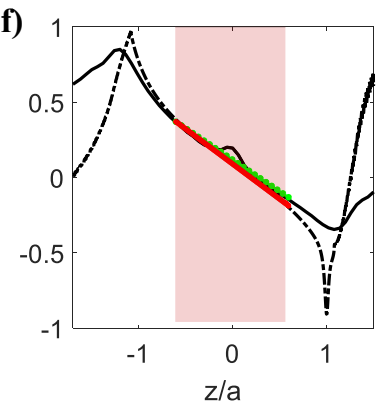

FIG. 18. Plots of trapping force against normalized axial direction using GO 3D distribution and EMT under both cw (top panel) and pulsed (bottom panel) excitation at $1 \mathrm{~mW}$ average power for fixed NA $=1.4$. Highlighted regime indicates the change in the nature of curve near the focus with increasing particle size.

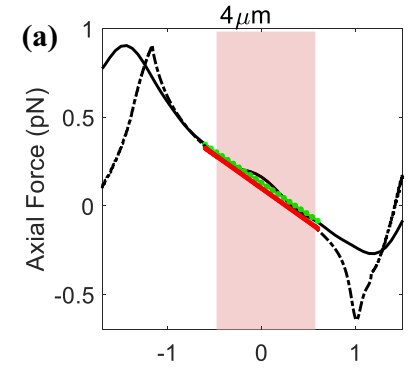

(d)
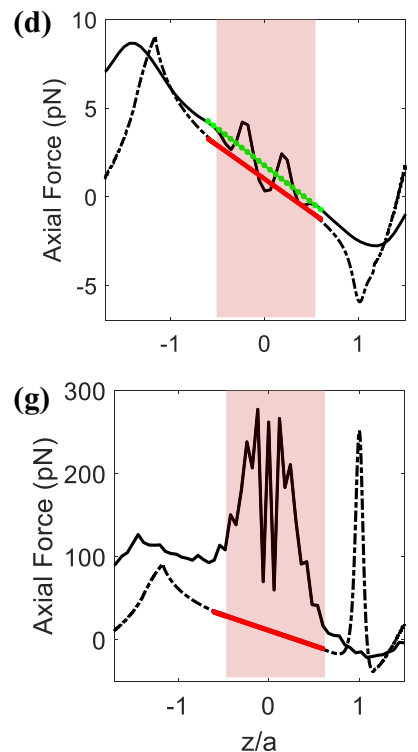

(b)

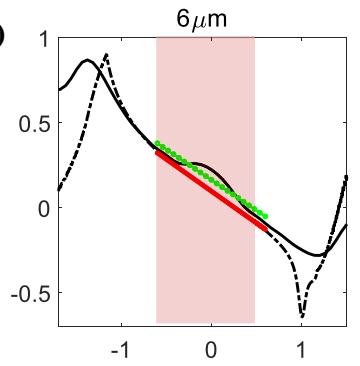

(e)

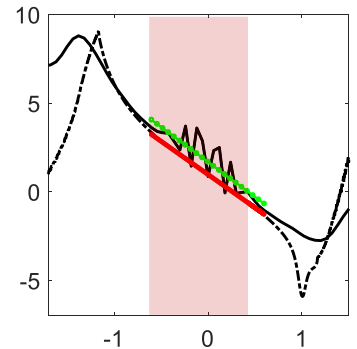

(h)

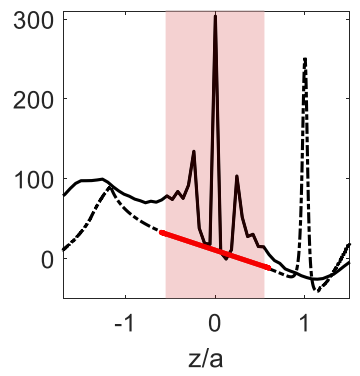

(c)

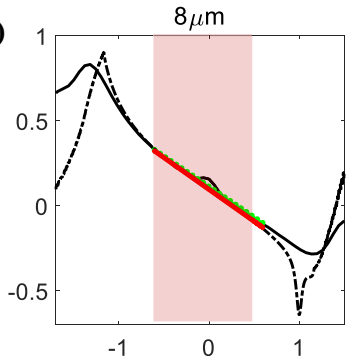

(f)

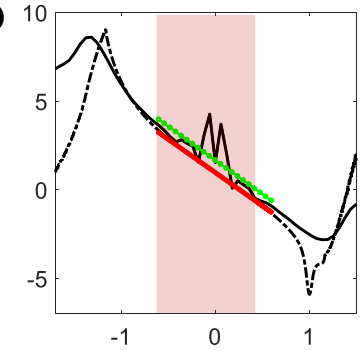

(i)

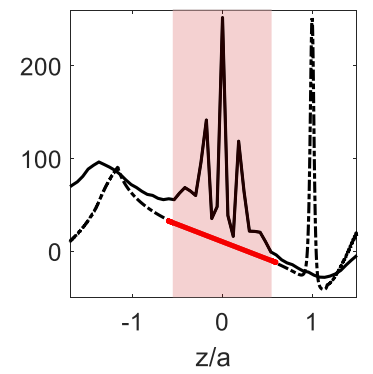

FIG. 19. Plots of trapping force against normalized axial direction using GO 3D distribution and EMT under pulsed excitation at different average power (top panel: $1 \mathrm{~mW}$; middle panel: $10 \mathrm{~mW}$; bottom panel: $100 \mathrm{~mW}$ ) for fixed $\mathrm{NA}=1.3$. Highlighted regime indicates the change in the nature of curve near the focus with increasing the particle size and average power. 
(a)

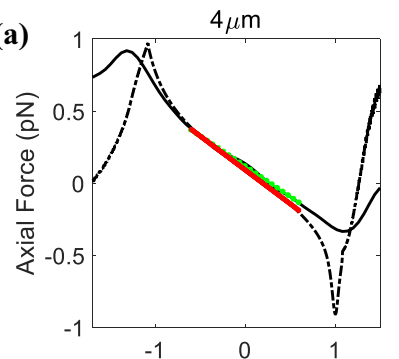

(d)
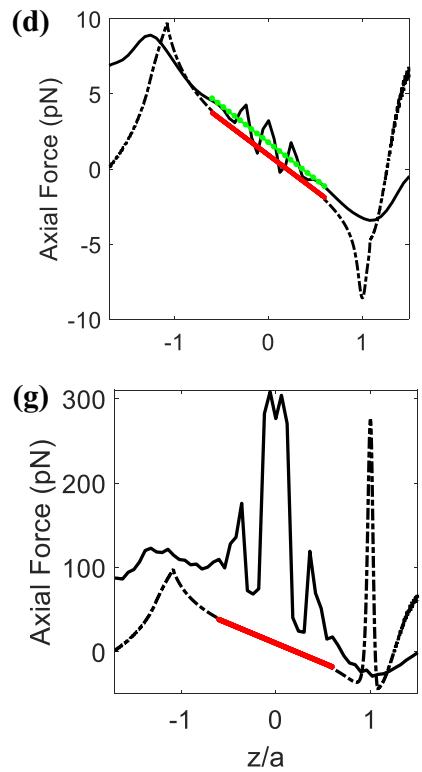

(b)

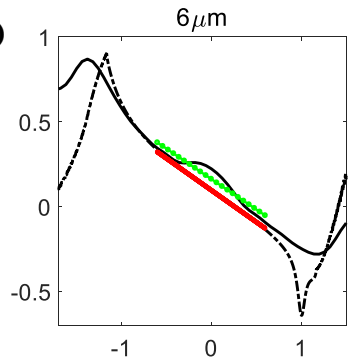

(e)

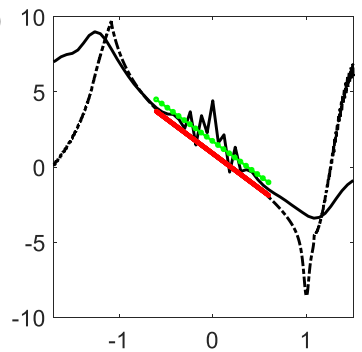

(h)

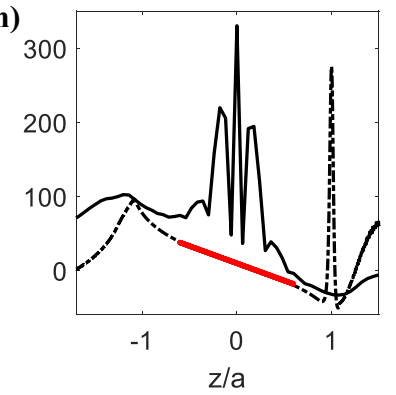

(c)

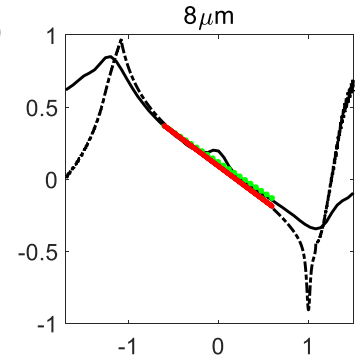

(f)

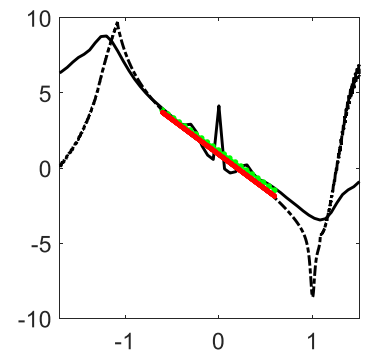

(i)

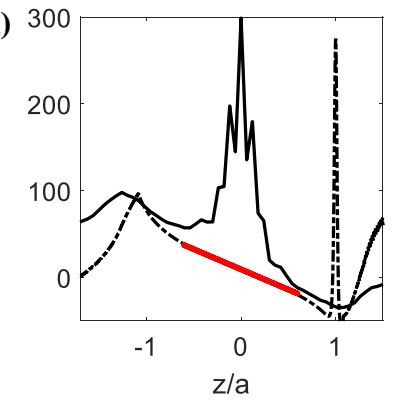

FIG. 20. Plots of trapping force against normalized axial direction using GO 3D distribution and EMT under pulsed excitation at different average power (top panel: $1 \mathrm{~mW}$; middle panel: $10 \mathrm{~mW}$; bottom panel: $100 \mathrm{~mW}$ ) for fixed $\mathrm{NA}=1.4$.

curve at different average powers for different particle sizes for fixed NA 1.4 under pulsed excitation. An increasing effect of nonlinearity can be seen with increasing power near the focus. Under pulsed excitation, MSCs are strongly dependent upon $a$ and $M$. If we compare cw excitation (Tables IV-VI) and pulsed excitation (Tables VII-IX), there is no significant change in $F_{\max }, F_{\min }$, and $k_{\text {stiffness. }}$. This is because nonlinear RI does not lead to a quantitative change in overall RI, but it does contribute qualitatively (through $M=\frac{n_{0}^{p}+n_{2}^{p} \times I(z)}{n_{0}^{w}}$ dependency

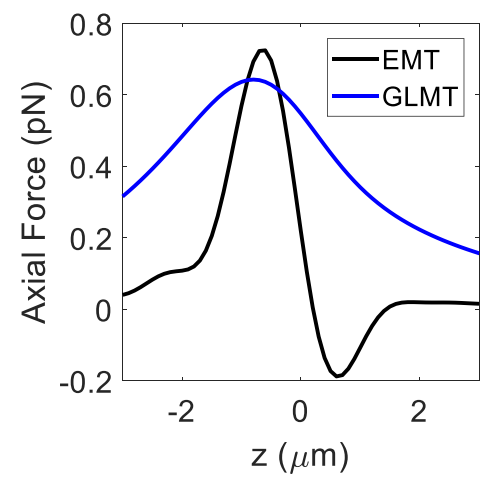

FIG. 21. Plots of trapping force against axial direction using GLMT and EMT under cw excitation for $0.4 \mu \mathrm{m}$ particle size at 1 $\mathrm{mW}$ average power for fixed $\mathrm{NA}=1.3$. on $z$ ) which results in changing the nature of the force curve near the focus. At high average power, the nonlinear RI term contributes significantly to overall RI, where a drastic change in the nature of the force curves is observed, as shown in Figs. 19 and 20. Please note that calculating $k_{\text {stiffness }}$ at higher average power under pulsed excitation is not appropriate by fitting it to a linear fit but we have fitted it just to compare the values with cw excitation. In EMT, for a fixed average power and NA, an increase in the particle size leads to a decrease in
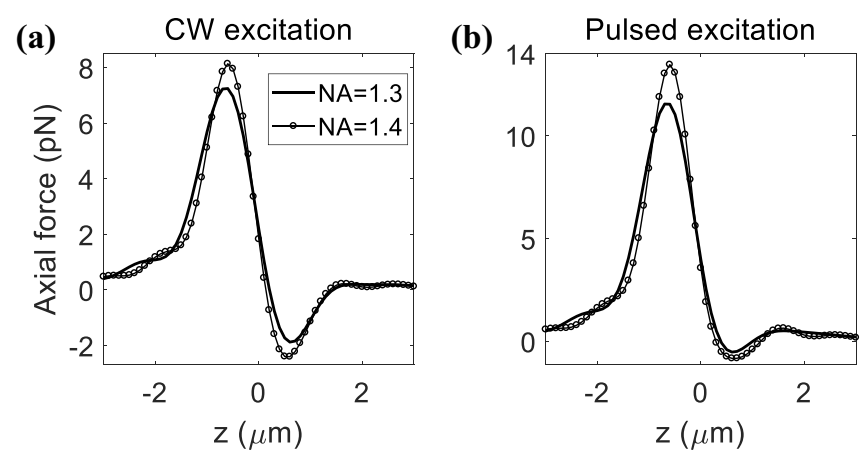

FIG. 22. Plots of trapping force against axial direction using GLMT and EMT under both cw (a) and pulsed (b) excitation for $0.4 \mu \mathrm{m}$ particle size at $10 \mathrm{~mW}$ average power. 
TABLE XI. The values of $F_{\max }$ under both cw and pulsed excitation at $10 \mathrm{~mW}$ average power for EMT.

\begin{tabular}{lcc}
\hline \hline NA & cw excitation & Pulsed excitation \\
\hline 1.3 & 7.25 & 11.55 \\
1.4 & 8.14 & 13.47 \\
\hline \hline
\end{tabular}

$k_{\text {stiffness }}$ while increasing NA leads to an increase in the $k_{\text {stiffness }}$ for fixed particle size and average power. Quite interestingly, the total force exhibits Mie interference behavior near the focus, and the amplitude of Mie interference increases with average power for fixed particle size. For a fixed power and $\mathrm{NA}=1.4$, the magnitude of Mie interference increases as compared to NA $=1.3$ (Fig. 20). However, such effects are not observed for the GO 3D distribution. In Table X, $k_{\text {stiffness }}$ increases almost linearly with increasing average power for the GO 3D distribution, whereas EMT gives nonlinear variation with average power, but at $100 \mathrm{~mW}$ average power, nonlinear effects dominate significantly and we could not measure the $k_{\text {stiffness }}$ appropriately.

\section{Mie regime}

For the Mie regime, the trapping force comparison is done for EMT and GLMT. Hereafter in the Mie regime, the black curve corresponds to the EMT, and the blue curve corresponds to the GLMT unless otherwise mentioned.

\section{a. cw excitation}

Both theories show a difference in magnitude as well as in the nature of the force curve, as shown in Fig. 21. From here, it is evident that the GLMT approximation is not a valid theory for the Mie regime. Hence, further analysis in the Mie regime is performed using EMT.
TABLE XII. The values of $k_{\text {stiffness }}\left(\frac{\mathrm{pN}}{\mu \mathrm{m}}\right)$ under both $\mathrm{cw}$ and pulsed excitation at $10 \mathrm{~mW}$ average power for EMT.

\begin{tabular}{lcc}
\hline \hline NA & cw excitation & Pulsed excitation \\
\hline 1.3 & 10.83 & 14.94 \\
1.4 & 13.85 & 19.84 \\
\hline \hline
\end{tabular}

Later on, the force calculations are done at $10 \mathrm{~mW}$ average power, to see the presence of the Mie interference structure while taking nonlinearity into account. Under $\mathrm{cw}$ excitation, no such behavior is present for the $0.4-\mu \mathrm{m}$ particle, as shown in Fig. 22(a).

\section{b. Pulsed excitation}

Under pulsed excitation, there is an overall change in $F_{\max }$ and $F_{\min }$ as compared to cw excitation at similar conditions which is quantitatively listed in Table XI and shown in Fig. 22(b). More appropriate evidence is reflected from $k_{\text {stiffness }}$ values, listed in Table XII. For fixed NA and particle size, $k_{\text {stiffness }}$ of $\mathrm{cW}$ excitation is less than pulsed excitation under similar conditions which implies that pulsed excitation gives better trapping efficiency than cw excitation. As we decrease the particle size, the particle experiences an impulsive force due to single pulses, hence providing more efficient trapping.

\section{Phase space trajectories}

The phase space trajectories are calculated for $40 \mathrm{~nm}$ (Fig. 23), $0.4 \mu \mathrm{m}$ (Fig. 24), and $4 \mu \mathrm{m}$ (Fig. 25) particle size at different average power under both $\mathrm{cw}$ and pulsed excitations. To calculate the phase space trajectories, we use the mass of
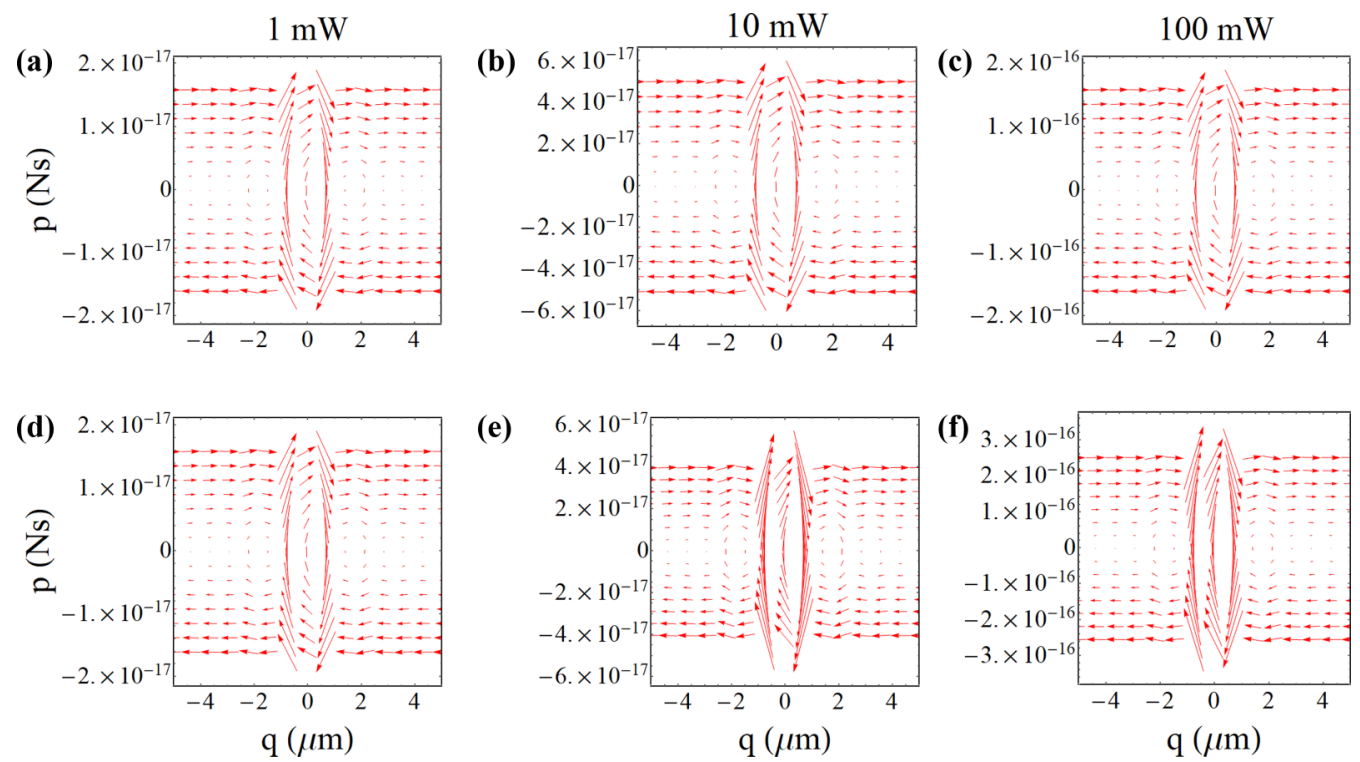

(e)
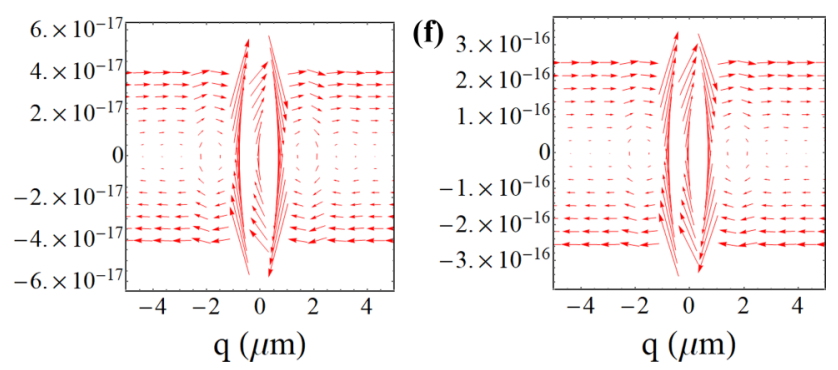

FIG. 23. Phase space trajectories of a single particle inside an optical trap for $40 \mathrm{~nm}$ particle at 1, 10, and $100 \mathrm{~mW}$ average power under both (a)-(c) cw and (d)-(f) pulsed excitation. 

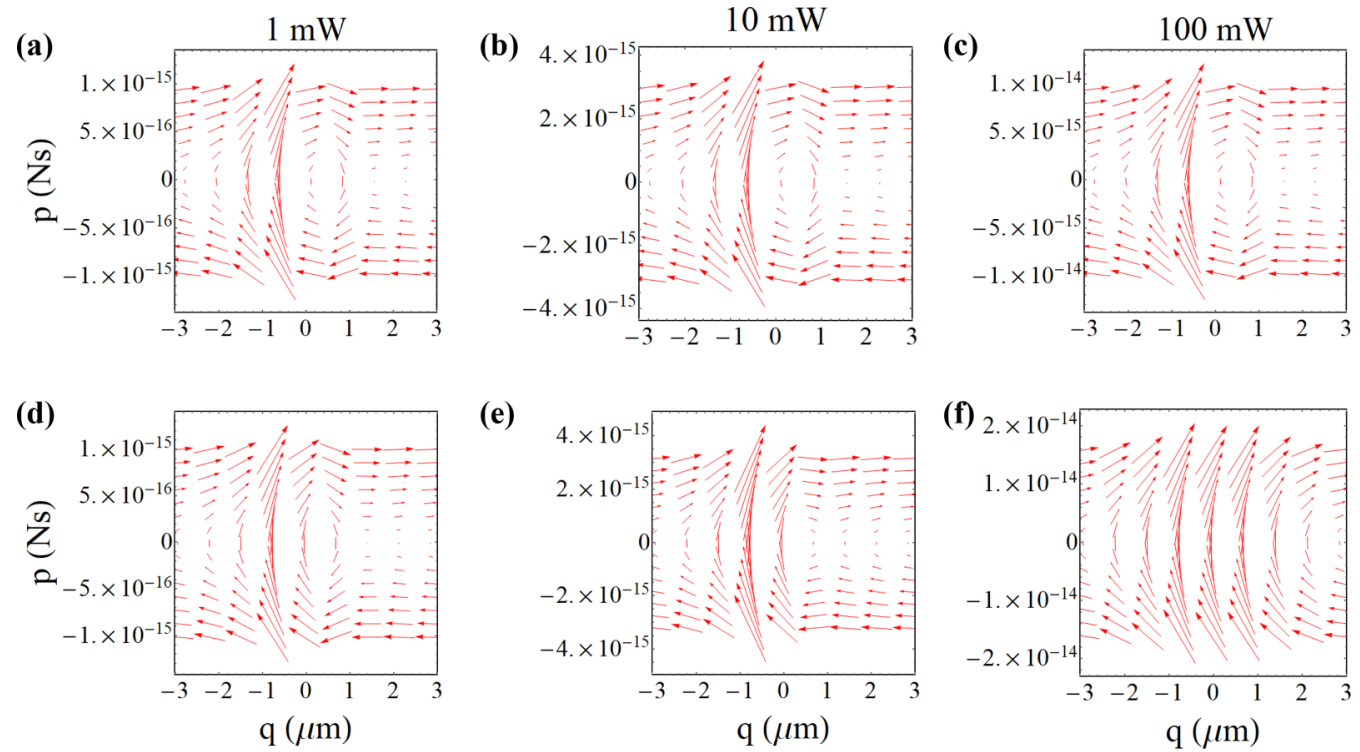

(e)

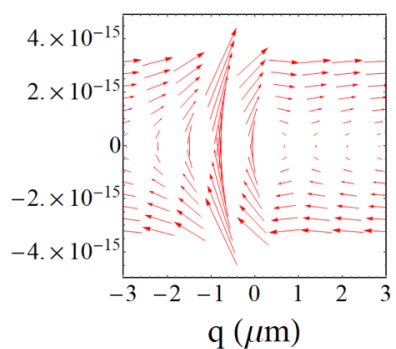

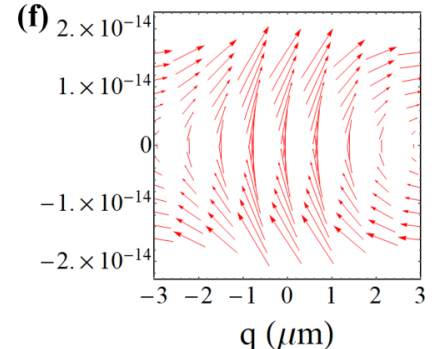

FIG. 24. Phase space trajectories of a single particle inside an optical trap for $0.4 \mu \mathrm{m}$ particle at 1,10 , and $100 \mathrm{~mW}$ average power under both (a)-(c) cw and (d)-(f) pulsed excitation.

single particles as $1 \times 10^{-19} \mathrm{~kg}, 1 \times 10^{-18} \mathrm{~kg}$, and $1 \times$ $10^{-17} \mathrm{~kg}$ for $40 \mathrm{~nm}, 0.4 \mu \mathrm{m}$, and $4 \mu \mathrm{m}$, respectively. It can be seen that with increasing average power, the effect of optical nonlinearity increases under pulsed excitation as compared to cw excitation. For 400-nm particles, the trap becomes completely unbound at $100 \mathrm{~mW}$ average power which implies that no stable trapping will be observed under specified conditions.
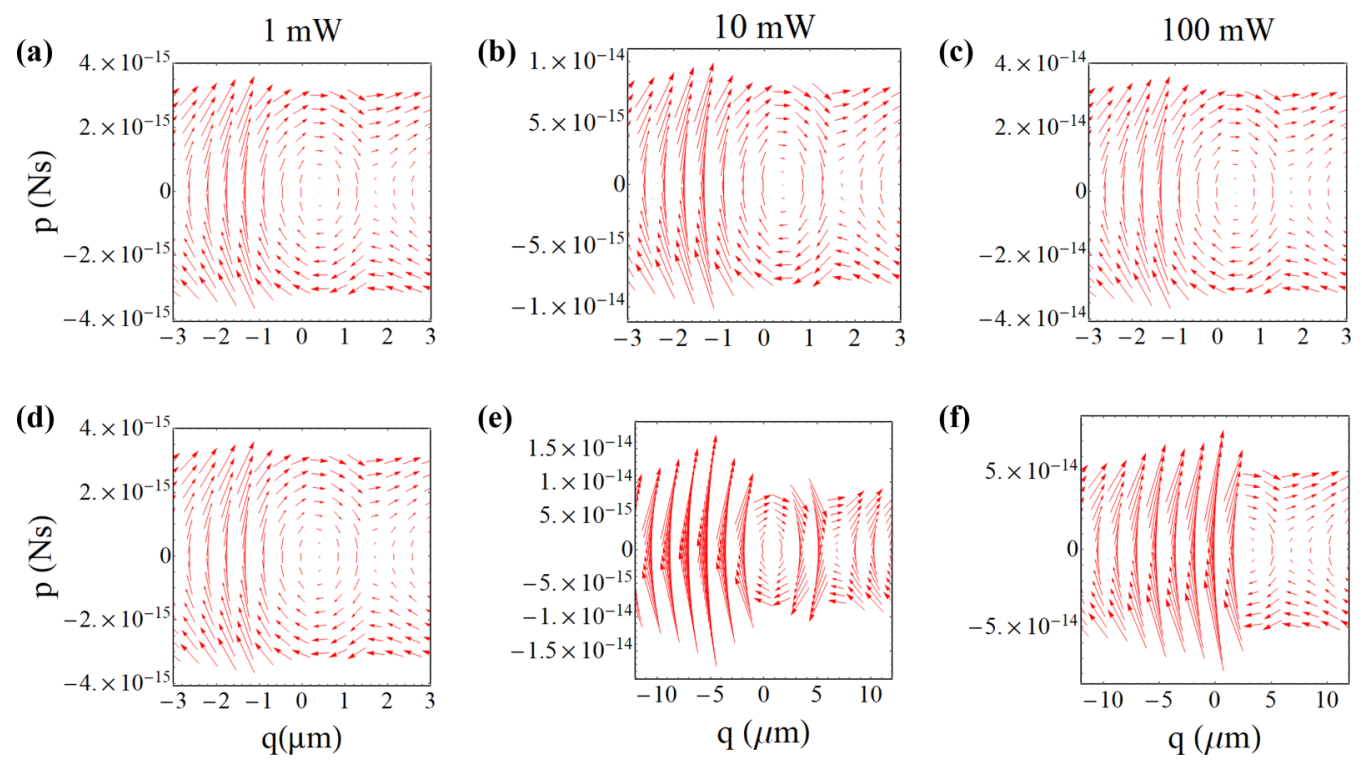

FIG. 25. Phase space trajectories of a single particle inside an optical trap for $4 \mu \mathrm{m}$ particle at 1,10 , and $100 \mathrm{~mW}$ average power under both (a)-(c) cw and (d)-(f) pulsed excitation. 
[1] A. Ashkin, Acceleration and Trapping of Particles by Radiation Pressure, Phys. Rev. Lett. 24, 156 (1970).

[2] A. Ashkin and J. M. Dziedzic, Trapping and manipulation of viruses and bacteria, Science 235, 1517 (1987).

[3] A. Ashkin, K. Schutze, J. M. Dsiedzic, U. Euteneuer, and M. Schliwa, Force generation of organelle transport measured in vivo by an infrared laser trap, Nature (London) 348, 346 (1990).

[4] K. Svoboda and S. M. Block, Biological application of optical forces, Annu. Rev. Biophys. Biomol. Struct. 23, 247 (1994).

[5] A. Ashkin, Optical Trapping and Manipulation of Neutral Particles Using Lasers: A Reprint Volume with Commentaries (World Scientific, Singapore, 2006).

[6] K. C. Neuman and S. M. Block, Optical trapping, Rev. Sci. Instrum. 75, 2787 (2004).

[7] J. R. Moffitt, Y. R. Chemla, S. B. Smith, and C. Bustamante, Recent advances in optical tweezers, Annu. Rev. Biochem. 77, 205 (2008).

[8] L. Pan, A. Ishikawa, and N. Tamai, Detection of optical trapping of CdTe quantum dots by two-photon-induced luminescence, Phys. Rev. B 75, 161305(R) (2007).

[9] J. C. Shane, M. Mazilu, W. M. Lee, and K. Dholakia, Effect of pulse temporal shape on optical trapping and impulse transfer using ultrashort pulsed lasers, Opt. Express 18, 7554 (2010).

[10] A. Usman, W-Y. Chiang, and H. Masuhara, Optical trapping and polarization-controlled scattering of dielectric spherical nanoparticles by femtosecond laser pulses, J. Photochem. Photobiol. A: Chem. 234, 83 (2012).

[11] A. Usman, W.-Y. Chiang, and H. Masuhara, Optical trapping of nanoparticles by ultrashort laser pulses, Sci. Prog. 96, 1 (2013).

[12] W.-Y. Chiang, A. Usman, and H. Masuhara, Femtosecond pulse-width dependent trapping and directional ejection dynamics of dielectric nanoparticles, J. Phys. Chem. C 117, 19182 (2013).

[13] W.-Y. Chiang, T. Okuhata, A. Usman, N. Tamai, and H. Masuhara, Efficient optical trapping of CdTe quantum dots by femtosecond laser pulses, J. Phys. Chem. B 118, 14010 (2014).

[14] A. Kittiravechote, A. Usman, H. Masuhara, and I. Liau, Enhanced optical confinement of dielectric nanoparticles by two-photon resonance transition, RSC Adv. 7, 42606 (2017).

[15] B. Agate, C. T. A. Brown, W. Sibbett, and K. Dholakia, Femtosecond optical tweezers for in-situ control of two-photon fluorescence, Opt. Express 12, 3011 (2004).

[16] L. G. Wang and C. L. Zhao, Dynamic radiation force of a pulsed Gaussian beam acting on a Rayleigh dielectric sphere, Opt. Express 15, 10615 (2007).

[17] L. G. Wang and H. S. Chai, Revisit on dynamic radiation forces induced by pulsed Gaussian beams, Opt. Express 19, 14389 (2011).

[18] T.-H. Liu, W.-Y. Chiang, A. Usman, and H. Masuhara, Optical trapping dynamics of a single polystyrene sphere: Continuous wave versus femtosecond lasers, J. Phys. Chem. C 120, 2392 (2016).

[19] A. Devi and A. K. De, Simultaneous detection of twophoton fluorescence and backscatter of optical trapping of dielectric nanoparticles under femtosecond pulsed excitation, J. Nanophotonics 13, 020501 (2019).
[20] A. Devi, S. Yadav, and A. K. De, Dynamics of a dielectric microsphere inside a nonlinear laser trap, Appl. Phys. Lett. 117, 161102 (2020).

[21] J. L. Deng, Q. Wei, Y. Z. Wang, and Y. Q. Li, Numerical modeling of optical levitation and trapping of the stuck particles with a pulsed optical tweezers, Opt. Express 13, 3673 (2005).

[22] A. A. Ambardekar and Y. Q. Li, Optical levitation and manipulation of stuck particles with pulsed optical tweezers, Opt. Lett. 30, 1797 (2005).

[23] A. Devi and A. K. De, Theoretical investigation on optical Kerr effect in femtosecond laser trapping of dielectric microparticles, J. Opt. 19, 065504 (2017).

[24] A. Devi and A. K. De, Alternate theoretical formulation of optical force on a dielectric sphere in the ray optics limit, J. Opt. Soc. Am. B 35, 244 (2018).

[25] D. Roy, D. Goswami, and A. K. De, Exploring the physics of efficient optical trapping of dielectric nanoparticles with ultrafast pulsed excitation, Appl. Opt. 54, 7002 (2015).

[26] A. Devi and A. K. De, Theoretical investigation on nonlinear optical effect in laser trapping of dielectric nanoparticles with ultrafast pulsed excitation, Opt. Express 24, 21485 (2016).

[27] A. Devi and A. K. De, Theoretical estimation of nonlinear optical force on dielectric spherical particles of arbitrary size under femtosecond pulsed excitation, Phys. Rev. A 96, 023856 (2017).

[28] Y. Jiang, T. Narushima, and H. Okamoto, Nonlinear optical effects in trapping nanoparticles with femtosecond pulses, Nat. Phys. 6, 1005 (2010).

[29] A. Devi, S. S. Nair, and A. K. De, Disappearance and reappearance of optical trap for silver nanoparticles under femtosecond pulsed excitation: A theoretical investigation, Europhys. Lett. 126, 28002 (2019)

[30] S. Yadav, A. Devi, and A. K. De, Synergistic effect of Fanoresonance and optical nonlinearity in laser trapping of silver nanoparticles, Phys. Rev. A 102, 043511 (2020).

[31] A. Devi and A. K. De, Harnessing optical nonlinearity to control reversal of trapping force: A theoretical investigation, J. Opt. 21, 065502 (2019).

[32] A. Devi and A. K. De, Generalized Lorentz-Mie theory for the reversal of the optical force in a nonlinear laser trap, Phys. Rev. A 102, 023509 (2020).

[33] P. A. Maia Netro and H. M. Nussenzveig, Theory of optical tweezer, Europhys. Lett. 50, 702 (2000).

[34] G. Gouesbet and G. Grehan, Generalized Lorentz-Mie Theory (Springer, Berlin, 2011).

[35] C. F. Bohren and D. R. Huffman, Absorption and Scattering of Light by Small Particles (Wiley, Germany, 2008).

[36] A. R. Edmonds, Angular Momentum in Quantum Mechanics (Princeton University Press, Princeton, 1957).

[37] M. V. Rybin, K. B. Samusev, I. S. Sinev, G. Semouchin, E. Semouchkina, Y. S. Kivshar, and M. F. Limonov, Mie scattering as a cascade of Fano resonances, Opt. Express 21, 30107 (2013).

[38] X. Kong and G. Xiao, Fano resonance in high-permittivity dielectric spheres, J. Opt. Soc. Am. A 33, 707 (2016).

[39] H. Chen, S. Liu, J. Zi, and Z. Lin, Fano resonance-induced negative optical scattering force on plasmonic nanoparticles, ACS Nano 9, 1926 (2015). 
[40] A. B. Stilgoe, T. A. Nieminen, G. Knoner, N. R. Heckenberg, and H. Runinsztein-Dunlop, The effect of Mie resonances on trapping in optical tweezers, Opt. Express 16, 15039 (2008).

[41] M. Lenzner, J. Kruger, S. Sartania, Z. Cheng, Ch. Spielmann, G. Mourou, W. Kautek, and F. Krausz, Femtosecond Optical Breakdown in Dielectrics, Phys. Rev. Lett. 80, 4076 (1998).

[42] Y. Harada and T. Asakura, Radiation forces on a dielectric sphere in the Rayleigh scattering regime, Opt. Commun. 124, 529 (1996).

[43] M. J. Weber, CRC Handbook of Optical Material (CRC, Boca Raton, FL, 2003), Vol. III.
[44] M. Jahja, On nonlinear optical constants of polystyrene, in International Symposium on Modern Optics and its Applications - 2011 (2011).

[45] X. Y. Hu, Q. H. Gong, Y. H. Liu, B. Cheng, and D. Zhang, All-optical switching of defect mode in two-dimensional nonlinear organic photonic crystals, Appl. Phys. Lett. 87, 23111 (2005).

[46] R. S. Dutra, N. B. Viana, P. A. Maia Neto, and H. M. Nussenzveig, Polarization effects in optical tweezers, J. Opt. A: Pure Appl. Opt. 9, S221 (2007).

[47] N. B. Viana, M. S. Rocha, O. N. Mesquita, A. Mazolli, P. A. Maia Neto, and H. M. Nussenzveig, Towards absolute calibration of optical tweezers, Phys. Rev. E 75, 021914 (2007). 